\title{
Optimality Program in Segment and String Graphs
}

\author{
Édouard Bonnet ${ }^{1}$ • Paweł Rzążewski² ${ }^{2}$
}

Received: 27 June 2018 / Accepted: 23 March 2019 / Published online: 6 April 2019

(c) The Author(s) 2019

\begin{abstract}
Planar graphs are known to allow subexponential algorithms running in time $2^{O(\sqrt{n})}$ or $2^{O(\sqrt{n} \log n)}$ for most of the paradigmatic problems, while the brute-force time $2^{\Theta(n)}$ is very likely to be asymptotically best on general graphs. Intrigued by an algorithm packing curves in $2^{O\left(n^{2 / 3} \log n\right)}$ by Fox and Pach (SODA'11), we investigate which problems have subexponential algorithms on the intersection graphs of curves (string graphs) or segments (segment intersection graphs) and which problems have no such algorithms under the Exponential Time Hypothesis (ETH). Among our results, we show that, quite surprisingly, 3-COLORING can also be solved in time $\left.2^{O\left(n^{2 / 3} \log O(1)\right.} n\right)$ on string graphs while an algorithm running in time $2^{o(n)}$ for 4-COLORING even on axisparallel segments (of unbounded length) would disprove the ETH. For 4-COLORING of unit segments, we show a weaker lower bound, excluding a $2^{o\left(n^{2 / 3}\right)}$ algorithm (under the ETH). The construction exploits the celebrated Erdôs-Szekeres theorem. The subexponential running time also carries over to Min FeEdBack Vertex SET, but not to Min Dominating Set and Min IndePendent Dominating SeT.
\end{abstract}

Keywords String graphs $\cdot$ Segment graphs $\cdot$ Subexponential algorithms $\cdot$ ETH

\section{Introduction}

Most combinatorial optimization and decision problems admit subexponential algorithms when restricted to planar graphs. More precisely, they can be solved in time $2^{O(\sqrt{n})}$, or $2^{\tilde{O}(\sqrt{n})}$ on planar graphs with $n$ vertices, while under the ETH (Exponen-

The extended abstract of this paper was presented at WG 2018 [8].

Paweł Rzążewski

p.rzazewski@mini.pw.edu.pl

Édouard Bonnet

edouard.bonnet@dauphine.fr

1 LIP, ENS Lyon, Lyon, France

2 Faculty of Mathematics and Information Science, Warsaw University of Technology, Warsaw, Poland 
tial Time Hypothesis, which asserts that 3- SAT cannot be solved in subexponential time $[27,28])$ they do not admit an algorithm running in time $2^{o(n)}$ on general graphs. The former is due to the facts that planar graphs have treewidth $O(\sqrt{n})$ and that we have efficient algorithms parameterized by the treewidth tw of the graph, namely running in $2^{O(\mathrm{tw})} n^{O(1)}$, or $2^{\tilde{O}(\mathrm{tw})} n^{O(1)}$.

The so-called bidimensionality theory [14,16-18] pushes this square-root phenomenon further by yielding $2^{O(\sqrt{k})} n^{O(1)}$ algorithms where $k$ is the targeted size of a solution (think for example of the problems of finding a maximum independent set or a minimum dominating set of size $k$ ). In a nutshell, it exploits a deep structural result by Robertson, Seymour, and Thomas [41]: planar graphs with treewidth tw have a $\Theta(t w)$-by- $\Theta(t w)$ grid as a minor (i.e., any graph obtained by deleting vertices and edges, and contracting edges). Thus, if the presence of a large grid minor makes the problem trivial (as in, one can always answer yes or always answer no), then one only has to solve efficiently instances with low treewidth; which, as we noted, can often be done. The claimed running time is obtained by defining large grids as $\Theta(\sqrt{k})$-by$\Theta(\sqrt{k})$, since their absence as minors implies that the treewidth is in $O(\sqrt{k})$. The bidimensionality theory is also used to obtain approximation schemes and linear kernels and could be generalized to graphs with bounded genus and graphs excluding a fixed minor [15].

A natural line of research is to generalize or extend the subexponential (parameterized) algorithms to classes of graphs which do not fall into those categories. For geometric intersection graphs, the situation is much richer than for planar graphs. For instance, Marx and Pilipczuk already observed that packing problems (of the kind of MAX INDEPENDENT SET) are more broadly subject to subexponential algorithms - running typically in $n^{O(\sqrt{k})}$ - than covering problems (of the kind of Min Dominating SET) - for which $n^{O(k)}$ is often essentially optimal under the ETH [36,37].

We briefly survey the existing results in the design of subexponential algorithms on geometric intersection graphs. A prominent role is played by intersection graph of families of fat objects, i.e., objects for which the aspect ratio (their length divided by their width) is bounded. We highlight that fat objects, and in particular disks and squares, often allow faster algorithms and the so-called square-root phenomenon. As we will see, subexponential algorithms are less frequent on intersection graphs of curves and segments but nevertheless present such as exemplified by MAX INDEPENDent Set, 3-Coloring, Min Feedback Vertex Set, and MaXimum Induced MATCHING.

\subsection{Subexponential Algorithms on Geometric Intersection Graphs}

By a ply of a family of geometric objects we denote the maximum number of objects covering a single point. Smith and Wormald show that for any collection of $n$ convex fat objects with ply $p$ there is a balanced separator of size $O(\sqrt{n p})$ [45]. This leads to subexponential algorithms when the ply is constant, or in general for problems becoming trivial when the ply is too large, such as k-COLORING. The $2^{\tilde{O}(\sqrt{n k})}$-time 
algorithm that this win-win provides for coloring $n$ fat objects, say disks, with $k$ colors is shown essentially optimal under the ETH by Biró et al. [5].

A next step may consist of designing $\mathrm{FPT}^{1}$ or $\mathrm{XP}^{2}$ algorithms where the dependency in the parameter is subexponential (for problems of the form "find $k$ vertices such that..."). Using a shifting argument à la Baker [4], Alber and Fiala obtain a $n^{O(\sqrt{k})}$ time algorithm to decide if one can find $k$ disjoint unit disks or squares among $n$ [3]. Marx and Pilipczuk generalize this result to packing $k$ disjoint polygons among $n$ in the same time [36,37]. Their approach is based on guessing a small separator in the medial axis (i.e., the Voronoi diagram of polygons) of a supposed solution, as suggested by Adamaszek and Wiese and Har-Peled to obtain QPTAS for geometric packing problems [1,2,26].

Marx showed that MaX IndePendent Set and Min Dominating Set in the intersection graphs of disks or squares are W[1]-hard, and therefore unlikely to be FPT [35]. Those reductions also show that the $n^{O(\sqrt{k})}$ algorithms [36,37] are essentially optimal under the ETH. Fomin et al. [21] observed that unit disks of bounded degree have treewidth $O(\sqrt{n})$ and used this fact to extend bidimensionality to unit disk graphs for a handful of problems. Recently, a superset of the previous authors gave $2^{O(\sqrt{k})} n^{O(1)}$-time algorithms for $k$-FEEDBACK VERTEX SET, $k$ - PATH, $k$-CyCle, EXACT $k$-CYCLE [20].

\subsection{Non-fat Objects: Segments and Strings}

Segment intersection graphs (or segment graphs in short) are the intersection graphs of straight-line segments in the plane. They are called unit segments if all the segments of a representation share the same length. For a fixed integer $k, k$-DIR is defined as the set of intersection graphs of segments, each parallel to one of fixed $k$ directions. Strings graphs are the intersection graphs of simple curves in the plane. Those curves can be assumed polygonal without loss of generality. The vertices of the polygonal curves in a geometric representation are called geometric vertices not to confuse them with the actual vertices of the graph. As shown by Kratochvíl and Matoušek, there are string graphs with $n$ vertices, which require $2^{\Omega(n)}$ geometric vertices in any string representation with polygonal curves [31].

A systematic study of segment graphs and their subclasses was initiated by Kratochvíl and Matoušek [32]. It is interesting to point out that every planar graph is a segment graph, as shown by Chalopin and Gonçalves [13] (this was a long-standing conjecture by Scheinerman [44], see also a recent proof by Gonçalvez, Isenmann, and Pennarun [25]).

The class of string graphs is very general, as it includes split graphs (i.e., graphs whose vertices can be partitioned into two sets inducing a clique and an independent set), intersection graphs of bodies (i.e., compact shapes with non-empty interior), or incomparability graphs (i.e., graphs whose vertex set is given by the set of elements of a poset, and edges join elements that are incomparable).

\footnotetext{
1 With running time $f(k) n^{O(1)}$.

2 With running time $n^{f(k)}$.
} 
Biró et al. showed that even though coloring disks or more generally fat objects with a constant number of colors can be solved in $2^{\tilde{O}(\sqrt{n})}$ [5], 6-coloring axis-parallel segments (2- DIR) in time $2^{o(n)}$ would refute the ETH. This suggests that subexponential algorithms are less frequent on the intersection graphs of non-fat objects such as segments and strings. On the other hand, Fox and Pach presented a subexponential algorithm for MAX INDEPENDENT SET on string graphs [22]. Their approach uses a win-win strategy and is based on the existence of balanced separators in string graphs. Fox, Pach, and Tóth showed that string graphs with $m$ edges have balanced separators of size $O\left(\mathrm{~m}^{3 / 4} \log m\right)$, and conjectured that there is always a separator of size $O(\sqrt{m})$ [24]. Matoušek showed that string graphs admit a balanced separator of size $O(\sqrt{m} \log m)$ [39]. Finally, recently Lee improved the result of Matoušek, proving the conjecture.

Theorem 1 (Lee [33]) Every string graph with $m$ edges has a balanced separator of size $O(\sqrt{m})$. Moreover, it can be found in polynomial time, provided that the geometric representation is given.

Let us point out that this result generalizes the famous planar separator theorem by Lipton and Tarjan [34], as planar graphs are string graphs and the number of edges in a planar graph is linear in the number of vertices. This also shows that Theorem 1 is best possible (up to the constants), as the planar separator theorem is asymptotically tight.

\subsection{Our Contributions}

We show that the subexponential algorithm for MAX INDEPENDENT SET in string graphs by Fox and Pach [22], running in time $2^{\tilde{O}\left(n^{2 / 3}\right)}$, can be extended to 3-COLORING and Min Feedback Vertex Set. As in the algorithm of Fox and Pach, the central idea is a win-win: either the graph is rather sparse and the separator of Theorem 1 gives a speed-up, or the graph has a high-degree vertex (used for 3-COLORING) or a large biclique (used for MIN FEEDBACK VERTEX SET) and an efficient branching can be performed. Refining a lower bound of Biró et al. [5], we complement this former result by showing that for any $k \geqslant 4, k$-COLORING cannot be solved in $2^{o(n)}$ even on axis-parallel segments, unless the ETH fails. The reduction relies on having segment lengths with two different orders of magnitude. We therefore ask if unit segments could allow a faster algorithm for $k$-COLORING for $k \geqslant 4$. Under the ETH, we provide a stronger lower bound than the one for planar graphs (which refutes a running time $2^{o(\sqrt{n})}$ ) and show that unit segments cannot be $k$-colored in $2^{o\left(n^{2 / 3}\right)}$ for any $k \geqslant 4$. Our construction uses the fact, closely related to the famous Erdős-Szekeres [19] theorem, that any permutation on $n$ totally ordered elements can be partitioned into $O(\sqrt{n})$ monotone subsequences (see Knuth [29, Sec. 5.1.4]).

We then give tight ETH lower bounds for Min (CONNECTED) Dominating SeT and Min InDEPENDENT Dominating SeT on segment graphs and MAX CliQue on string graphs. For that, we design reductions whose number $n$ of produced segments is linear in $N+M$ from satisfiability problems with $N$ variables and $M$ clauses. Indeed, the sparsification lemma of Impagliazzo et al. [28] implies that those satisfiability 
Table 1 Upper and lower bounds for classical problems on string and segment graphs

\begin{tabular}{|c|c|c|}
\hline Problem & Upper bound & Lower bound \\
\hline MAX INDEPENDENT SET & $2^{\tilde{O}(\sqrt{n})} p^{O(1)}, 2^{\tilde{O}\left(n^{2 / 3}\right)}$ & $2^{\Omega(\sqrt{n})}$ \\
\hline 3-COLORING & $2^{\tilde{O}\left(n^{2 / 3}\right)}$ & $2^{\Omega(\sqrt{n})}$ \\
\hline$k$-COLORING for every $k \geqslant 4$ & $2^{O(n)}$ & $2^{\Omega(n)}($ even in 2-DIR $)$ \\
\hline$k$-COLORING for every $k \geqslant 4$ & $2^{O(n)}$ & $2^{\Omega\left(n^{2 / 3}\right)}$ in unit 3-DIR \\
\hline Min FEEDback VeRTEX Set & $2^{\tilde{O}\left(n^{2 / 3}\right)}$ & $2^{\Omega(\sqrt{n})}$ \\
\hline MAXIMUM INDUCED MATCHING & $2^{\tilde{O}\left(n^{2 / 3}\right)}$ & $2^{\Omega(\sqrt{n})}$ \\
\hline Min (CONNECTED) Dominating SET & $2^{O(n)}$ & $2^{\Omega(n)}$ \\
\hline Min INDEPENDENT Dominating SET & $2^{O(n)}$ & $2^{\Omega(n)}$ \\
\hline Max Clique & $2^{O(n)}$ & $2^{\Omega(n)}$ (on string graphs) \\
\hline
\end{tabular}

The upper bounds work on string graphs. The lower bounds are conditional on the ETH, and designed on segment graphs, unless stated otherwise. New results are indicated by bold emphasis. By $p$ we denote the number of geometric vertices if a geometric representation is given

problems are not solvable in $2^{o(N+M)}$ unless the ETH fails; which enables us to conclude that the problems are not solvable in $2^{o(n)}$ under the ETH, on graphs with $n$ vertices.

Although the NP-hardness of the mentioned problems is known for segment intersection graphs $[11,47]$, getting such linear reductions might be difficult.

For instance, while it is known that planar graphs are a subclass of segment intersection graphs [13], implying the NP-hardness of all the problems of Table 1 except $\mathrm{k}$-COLORING for $k \geqslant 4$ and MAX CliQUE, this fact does not serve our purpose since they can be solved in time $2^{O(\sqrt{n})}$ on planar graphs. The situation is an interesting intermediate between planar and general graphs. Our objects can intersect but we cannot afford crossover gadgets (at least not quadratically many). Certain intersections create unwanted edges, whose importance we have to tame. It is also noteworthy that segment/string graphs cannot be expanders since if they have constant degree, by Theorem 1, they have treewidth $\tilde{O}(\sqrt{n})$. Hence, we are deprived of the usual hardest instances.

\subsection{Geometric Representation and Robust Algorithms}

In case of graphs with geometric representations, it is important to distinguish between a graph itself (i.e., a pure abstract structure, for which we know that some geometric representation exists), and the representation itself. Note that this is not the case with planar graphs, as finding a plane embedding can be done in linear time [9].

Finding a segment or string representation of a graph was shown to be NP-hard by Kratochvíl [30], and Kratochvíl and Matoušek [32], respectively. However, it was very unclear if the problems are in NP (which is usually the trivial part of an NPcompleteness proof). As mentioned above, Kratochvíl and Matoušek [31] showed that some string graphs require a representation of exponential size, which proved 
that the simple idea of exhaustively guessing the representation cannot work for this problem. Finally, the NP-membership of recognizing string graphs was proven by Schaefer et al. [42].

The story of recognizing segment graphs is even more interesting. On the first sight, the situation seems simpler than for strings, as the number of geometric points in a segment representation is clearly polynomial in $n$. However, there are segment graphs, whose every segment representation requires points with coordinates doubly exponential in $n$, i.e., using $2^{\Omega(n)}$ digits (see Kratochvíl and Matoušek [32], and McDiarmid and Müller [40]). Finally, the problem was shown to be complete for the class $\exists \mathbb{R}$ (see Schaefer and Štefankovič [43]), i.e., the class of problems reducible in polynomial time to deciding if a given existential formula over the reals is true. This is a strong evidence that the problem is not in NP. For a nice exposition of the $\exists \mathbb{R}$-completeness proof, see Matoušek [38].

All this shows that a requirement of an explicit geometric representation of an input graph may be a serious drawback of an algorithm. We call an algorithm robust if it takes only an abstract structure like a graph as an input, and either computes the solution, or concludes (correctly) that the input graph does not belong to the desired class. On the one hand, our algorithms (see Sect. 2) are robust, but work slightly faster if the input is given along with the geometric representation. On the other hand, the lower bounds (see Sect. 3) hold even if the geometric representation is given explicitly.

\section{Upper Bounds}

Fox and Pach showed that, on string graphs, a maximum independent set can be computed in subexponential time:

Theorem 2 (Fox and Pach [22], Lee [33]) MAX INDEPENDENT SET can be solved in time $2^{O\left(n^{2 / 3} \log n\right)}$ in string graphs with $n$ vertices.

In their paper, they give a worse running time than the one claimed above. This is because they used the $O\left(m^{3 / 4} \log m\right)$ separator theorem [24], which has been recently improved to $O(\sqrt{m})$ [33]. The algorithm is a simple win-win argument. If there is a vertex with degree at least $n^{1 / 3}$, then either removing it or selecting it and removing its neighbors gives a branching $F(n) \leqslant F(n-1)+F\left(n-\left\lceil n^{1 / 3}\right\rceil-1\right)$. Otherwise, if all the vertices have degree smaller than $n^{1 / 3}$, the graph is rather sparse and the balanced separator of size $O(\sqrt{m})=O\left(n^{2 / 3}\right)$ provides an efficient divide-and-conquer. The threshold $n^{1 / 3}$ is computed so that it balances the running time of those two subroutines and gives the claimed overall asymptotic time.

This result was somewhat improved by Marx and Pilipczuk [36,37] based on an approach introduced by Adamaszek, Har-Peled, and Wiese [1]. However, their algorithm requires that the string graph is given with a representation by polygonal curves on a polynomial number of geometric vertices.

Theorem 3 (Marx and Pilipczuk [36]) MAX INDEPENDENT SET can be solved in time $2^{O(\sqrt{n} \log n)} p^{O(1)}$ in string graphs with $n$ vertices, where the strings are given as polygonal curves on a total of p geometric vertices. 
In a nutshell, the idea is to exhaustively guess a small balanced face-separator in the Voronoi diagram of a supposed (although not known) fixed solution, and solve recursively the two subinstances in the inside and outside of this separator.

Let us point out that since the complement of an independent set is a vertex cover, the algorithms from Theorems 2 and 3 can be used to solve the MIN VERTEX COVER problem within the same time bounds.

If the approach of Marx and Pilipczuk does not seem to generalize easily to coloring problems, the win-win of Fox and Pach can be transported to 3-COLORING with a bit more arguments. The algorithm even works for the more general LIST 3-COLORING (in List k-COLORING each vertex $v$ is equipped with a list $L(v) \subseteq[k]$ and we want to find a proper coloring, in which every vertex receives a color from its list).

Theorem 4 LIST 3-COLORING of a string graph with $n$ vertices can be decided in time $2^{O\left(n^{2 / 3} \log n\right)}$, even without geometric representation.

Proof Consider an instance $(G, L)$ of LIST 3-COLORING with $n$ vertices. Observe that without loss of generality we can assume that each list has two or three elements. Indeed, if there is a vertex with just one allowed color, we can fix this color and remove it from the list of each of its neighbors. Let $N$ be the sum of the lengths of the lists; clearly $2 n \leqslant N \leqslant 3 n$.

First, assume that $G$ has no vertex with degree larger than $n^{1 / 3}$, then the number $m$ of edges is $O\left(n^{4 / 3}\right)$. By Theorem $1, G$ has a balanced separator of size $O(\sqrt{m})=$ $O\left(n^{2 / 3}\right)$. We can find this separator in polynomial time, if the representation is given, or by exhaustive guessing in time $n^{O\left(n^{2 / 3}\right)}=2^{O\left(n^{2 / 3} \log n\right)}$, without using a representation. Then we list all possible colorings of the separator and proceed with a standard divideand-conquer approach. The total time complexity of this step is $2^{O\left(n^{2 / 3} \log n\right)}$.

If there is a vertex $v$ of degree at least $n^{1 / 3}$, then one among the lists: $\{1,2\},\{1,3\},\{2,3\},\{1,2,3\}$ appears on at least $n^{1 / 3} / 4$ of its neighbors. Thus there are two colors (say, 1 and 2) that appear in lists of at least $n^{1 / 3} / 4$ of neighbors of $v$. Since the list of $v$ has size at least two, one of these colors (say 1) appears on the list of $v$. We branch into two possibilities: choosing the color 1 for $v$ (then we exclude 1 from the lists of all the neighbors of $v$ ), and not choosing 1 for $v$ (then we remove 1 from the list of $v$ ). The complexity $F$ of this step is given by the recursion

$$
\begin{aligned}
F(N) & \leqslant F\left(N-n^{1 / 3} / 4\right)+F(N-1) \leqslant F\left(N-N^{1 / 3} /\left(3^{1 / 3} \cdot 4\right)\right)+F(N-1) \\
& \leqslant F\left(N-N^{1 / 3} /\left(3^{1 / 3} \cdot 4\right)\right)+F\left(N-N^{1 / 3} /\left(3^{1 / 3} \cdot 4\right)\right)+F(N-2) \\
& \leqslant 3 \cdot F\left(N-N^{1 / 3} /\left(3^{1 / 3} \cdot 4\right)\right)+F(N-3) \leqslant \ldots \\
& \leqslant\left(N^{1 / 3} /\left(3^{1 / 3} \cdot 4\right)\right) \cdot F\left(N-N^{1 / 3} /\left(3^{1 / 3} \cdot 4\right)\right)+F\left(N-N^{1 / 3} /\left(3^{1 / 3} \cdot 4\right)\right) \\
& =\left(N^{1 / 3} /\left(3^{1 / 3} \cdot 4\right)+1\right) \cdot F\left(N-N^{1 / 3} /\left(3^{1 / 3} \cdot 4\right)\right) .
\end{aligned}
$$


This inequality is satisfied by

$$
F(N)=\left(N^{1 / 3} /\left(3^{1 / 3} \cdot 4\right)+1\right)^{\frac{N}{N^{1 / 3} /\left(3^{1 / 3} \cdot 4\right)}}=2^{O\left(N^{2 / 3} \log N\right)}=2^{O\left(n^{2 / 3} \cdot \log n\right)}
$$

Combining these two cases gives the claimed time complexity. Finally, observe that if the input graph is not a string graph, then the exhaustive search for a separator might fail, and then we can report a wrong input instance.

In Min FeEdback Vertex SET problem we ask for the minimum set of vertices, whose removal destroys all cycles in a graph. For this problem, there is no obvious subexponential branching on a high-degree vertex. Instead, we use the following theorem by Lee.

Theorem 5 (Lee [33]) There is a constant c such that for any $t \geqslant 1, K_{t, t}$-free string graphs on $n$ vertices have fewer than $c \cdot t \log t \cdot n$ edges.

It is worth mentioning that Fox and Pach [23, Theorem 5] obtained a slightly weaker result with $\log { }^{O(1)} t$ instead of $\log t$. Theorem 5 is the last tool we need to show the following.

Theorem 6 MIN FEEDBACK VERTEX SET on string graphs with $n$ vertices can be solved in time $2^{\tilde{O}\left(n^{2 / 3}\right)}$.

Proof The proof is similar to the proof of Theorem 4, but it involves a slight technical complication. We will solve a more general problem, where the input is a graph $G$, a set $\mathcal{C}_{1}$ of constraints of type disconnect $(u, v)$, and another $\operatorname{set} \mathcal{C}_{2}$ of constraints of type stays $(v)$, where $u, v$ are vertices of $G$. We ask for a minimum feedback vertex set $X$ of $G$, such that for every constraint disconnect $(u, v)$, the vertices $u$ and $v$ are in different connected components of $G-X$, and for every constraint stays $(v)$ we have $v \notin X$. The algorithm is recursive, the constraints $\mathcal{C}_{1}$ and $\mathcal{C}_{2}$ are checked at the leaves of the recursion tree. Clearly, if $\mathcal{C}_{1}=\mathcal{C}_{2}=\emptyset$, then we just ask for the minimum feedback vertex set.

If $G$ has fewer than $c / 3 \cdot n^{4 / 3} \log n$ edges (where $c$ is a constant from Theorem 5), then by Theorem 1 there is a balanced separator $S$ of size $O(\sqrt{m})=\tilde{O}\left(n^{2 / 3} \log ^{1 / 2} n\right)$, we can find it in time $n^{O\left(n^{2 / 3}\right)}=2^{O\left(n^{2 / 3} \log ^{3 / 2} n\right)}$ by exhaustive search or in polynomial time, if the geometric representation is given. We partition the vertices of $G-S$ into sets $V_{1}, V_{2}$, such that $V_{1}, V_{2} \leqslant c^{\prime} \cdot n$ (for a constant $c^{\prime}$ ) and $S$ separates $V_{1}$ from $V_{2}$; such sets exist since $S$ is a balanced separator. We will exhaustively guess the intersection $I^{\prime}$ of a fixed minimum solution with $S$, taking into consideration the current constraints $\mathcal{C}_{2}$ (this represents at most $2^{|S|}=2^{\tilde{O}\left(n^{2 / 3}\right)}$ possibilities), introduce the new constraints $\operatorname{stays}(v)$ for every $v \in S \backslash I^{\prime}$, and solve the problem independently in $G_{1}:=G\left[V_{1} \cup S \backslash I\right]$ and $G_{2}:=G\left[V_{2} \cup S \backslash I\right]$.

However, note that there might be cycles in $G$ that are not fully contained in $V_{1} \cup S$ or in $V_{2} \cup S$ and the straightforward approach discussed above would not destroy them. Let us call such cycles essential. For each essential cycle $C$, and for $i=1,2$, we call $i$-subpath of $C$ a subpath of $C$, whose endvertices are in $S$, and inner vertices are in $V_{i}$. To destroy $C$, we must disconnect the endvertices of some $i$-subpath of $C$. 
We ensure this by introducing appropriate separation constraints. For every partition $\Pi=\left(S_{1}, S_{2}, \ldots, S_{k}\right)$ of $S \backslash I$, we run the algorithm recursively in each graph $G_{i}$ with additional constraints disconnect $(u, v)$ for every $u, v \in S$, such that $u$ and $v$ are in different parts of $\Pi$. The number of partitions of $S \backslash I$ is given by the Bell number of $|S \backslash I|$, which is upper-bounded by $|S|^{|S|}=2^{|S| \log |S|}=2^{\tilde{O}\left(n^{2 / 3}\right)}$. This gives us a total of $2^{\tilde{O}\left(n^{2 / 3}\right)}$ recursive calls at each level of the recursion tree. It is sufficient to only consider those connectivity patterns since being connected is a transitive relation: if $u$ and $v$, and $v$ and $w$ stay connected in $G_{i}$, then $u$ and $w$ also stay connected. We combine solutions in $G_{1}$ and $G_{2}$ which agree on the subset $I \subseteq S$, and such that the essential cycles cannot survive. It is known and relatively easy to see that this happens exactly when the partitions $\Pi^{1}=\left(S_{1}^{1}, S_{2}^{1}, \ldots, S_{k_{1}}^{1}\right)$ for $G_{1}$ and $\Pi^{2}=\left(S_{1}^{2}, S_{2}^{2}, \ldots, S_{k_{2}}^{2}\right)$ for $G_{2}$ are such that for each pair $(i, j),\left|S_{i}^{1} \cup S_{j}^{2}\right| \leqslant 1$ and the bipartite intersection graph (with an edge between $S_{i}^{1}$ and $S_{j}^{2}$ iff they have non-empty intersection) is a forest (see e.g., [6,7]). This way, for every connectivity pattern in $S \backslash I$, we will find the minimum feedback vertex set respecting this pattern. When we combine the solutions of both subproblems, we reject the ones that do not agree on $S$, and those with an essential cycle $C$ for which the endvertices of every $i$-subpath are not disconnected in $G_{i}$. This step has a total running time $2^{\tilde{O}\left(n^{2 / 3}\right)}$.

On the other hand, if $G$ has at least $c / 3 \cdot n^{4 / 3} \log n$ edges, by Theorem 5 , there is a subgraph of $G$ isomorphic to $K_{n^{1 / 3}, n^{1 / 3}}$. We can find it by exhaustive guessing in time $n^{2 n^{1 / 3}} \cdot \operatorname{poly}(n)=2^{\tilde{O}\left(n^{1 / 3}\right)}$. Observe that any feedback vertex set of $G$ must contain all but one vertex of one bipartition class of the biclique. This observation gives us a branching algorithm: we choose the vertex $v$ which is not necessarily included in the the solution in $2 n^{1 / 3}$ ways, then we remove all other vertices from the bipartition class of $v$, and proceed recursively. Note that $v$ might be still chosen to the solution in next steps. The complexity of this algorithm is given by the recursion

$$
F(n) \leqslant 2^{\tilde{O}\left(n^{1 / 3} \log n\right)}+2 n^{1 / 3} F\left(n-n^{1 / 3}+1\right) .
$$

Note that the depth of recursion is $O\left(n^{2 / 3}\right)$, so the inequality is solved by

$$
F(n)=\left(2 n^{1 / 3}\right)^{O\left(n^{2 / 3}\right)}=2^{O\left(n^{2 / 3} \log n\right)} .
$$

We also trim the branches which violate a constraint of $\mathcal{C}_{2}$. Branching does not introduce new constraints. In particular, the vertex which is not added to the solution (unlike all the other vertices of its bipartition class) might still be included in the solution at a later stage. Observe that the branching on a separator and the branching on a biclique produce instances of the same problem and can be done in an interleaved fashion.

Finally, if the exhaustive search for a separator or a biclique fails, then either we have reached a constant size, in which case the subproblem can be brute-forced, or we can correctly report that the input graph is not a string graph.

It is interesting to note that a similar approach works also for the MAXIMUM INDUCED MATCHING problem. 
Theorem 7 MAXIMUM INDUCED MATCHING on string graphs with $n$ vertices can be solved in time $2^{\tilde{O}\left(n^{2 / 3}\right)}$.

Proof Again, to ensure consistency of solutions found in recursive calls, we will solve a more general problem, where we are given a graph $G$ and a subset $X$ of its vertices, and we ask for a maximum induced matching which covers all vertices from $X$. Clearly, if $X=\emptyset$, then we just ask for a maximum induced matching. Whenever in the recursion a neighbor $u$ of a vertex $v \in X$ becomes matched to some vertex other than $v$, we can immediately terminate the current recursive call, as it will not produce a feasible solution.

If $G$ has fewer than $c / 3 \cdot n^{4 / 3} \log n$ edges (where $c$ is a constant from Theorem 5), then by Theorem 1 there is a balanced separator $S$ of size $O(\sqrt{m})=\tilde{O}\left(n^{2 / 3} \log ^{1 / 2} n\right)$, which can be found in time $2^{O\left(n^{2 / 3} \log ^{3 / 2} n\right)}$ by exhaustive search or in polynomial time if the geometric representation is given. We partition the vertices of $G-S$ into sets $V_{1}, V_{2}$, such that $V_{1}, V_{2} \leqslant c^{\prime} \cdot n$ (for a constant $c^{\prime}$ ) and $S$ separates $V_{1}$ from $V_{2}$. We guess the set $I \subseteq S$ of vertices covered by a fixed optimal solution, and for each vertex $v \in I$ we consider separately cases when the edge covering $v$ belongs to $G_{1}:=G\left[V_{1} \cup S\right]$ or to $G_{2}:=G\left[V_{2} \cup S\right]$ and solve the problem independently in $G_{1}$ and $G_{2}$. If a vertex $v$ is chosen to be covered by an edge from, say, $G_{1}$, then in the recursive call in $G_{2}$ we can remove $v$ and all its neighbors. This gives at most $2 \cdot 3^{|S|}=2^{\tilde{O}\left(n^{2 / 3}\right)}$ recursive calls and the total complexity of this step is $2^{\tilde{O}\left(n^{2 / 3}\right)}$.

In the other case, if the number of edges is at least $c / 3 \cdot n^{4 / 3} \log n$, then by Theorem 5 , $G$ contains a copy of the biclique $K_{n^{1 / 3}, n^{1 / 3}}$ as a (non-necessarily induced) subgraph. Let $A$ and $B$ be the bipartition classes of the biclique and consider some fixed solution $S$. Observe that if $S \cap(A \cup B) \neq \emptyset$, then either (a) $S$ contains an edge $u v$ where $u \in A$ and $v \in B$, and no other vertex of $A \cup B$ is covered, or (b) $V(S)$ contains a vertex $u$ from, say, $A$, and no vertex of $B$ (or the other way around). In case (a) the edge $u v$ can be chosen in at most $n^{2 / 3}$ ways and we can safely remove all vertices of the biclique, and in case (b) the vertex $u$ can be chosen in at most $2 n^{1 / 3}$ ways and we remove all vertices of the other bipartition class. Finally, if $S \cap(A \cup B)=\emptyset$, we remove all vertices of the biclique. Thus we obtain a branching algorithm, whose complexity is given by a recursion:

$$
\begin{aligned}
F(n) & \leqslant 2^{\tilde{O}\left(n^{2 / 3}\right)}+\underbrace{F\left(n-2 n^{1 / 3}\right)}_{S \cap(A \cup B)=\emptyset}+\underbrace{n^{2 / 3} F\left(n-2 n^{1 / 3}\right)}_{\text {case (a) }}+\underbrace{2 n^{1 / 3} F\left(n-n^{1 / 3}\right)}_{\text {case (b) }} \\
& \leqslant 2^{\tilde{O}\left(n^{2 / 3}\right)}+2 n^{2 / 3} F\left(n-n^{1 / 3}\right) .
\end{aligned}
$$

Note that in case (b) we exhaustively guess the vertex $v$ from $V(S)$ and add it to $X$ in the recursive call. The inequality above is solved in a way analogous to inequality (1). The complexity of this step is again bounded by $2^{\tilde{O}\left(n^{2 / 3}\right)}$, and so is the running time of the whole algorithm.

We observe that the approach of Theorem 7 can be further generalized to find induced packings of copies of any fixed graph $H$. In the MAXIMUM INDUCED $H$ PACKING problem we are given a graph $G$ and ask for a maximum subset $X$ of vertices 
of $G$, such that $G[X]$ consists of disjoint copies of $H$. Observe that if $H=K_{2}$, then MAXIMUM INDUCED $H$-PACKING is precisely MAXIMUM INDUCED MATCHING. Even more generally, we can consider a problem of MAXIMUM INDUCED $\mathcal{H}$ - PACKING for a fixed finite set of graphs $\mathcal{H}$. In this problem we are given a graph $G$, and we look for the largest set $X \subseteq V(G)$, such that each connected component of $G[X]$ is isomorphic to some graph from $\mathcal{H}$.

The general idea is exactly the same as in the proof of Theorem 7, but the technical details are significantly more complicated. Recall that in the MAXIMUM INDUCED MATCHING problem we needed to exhaustively guess the intersection $I$ of an optimal solution $X$ with the balanced separator $S$, and for each $v \in I$ we considered the cases that $v$ is matched to a vertex of $G_{1}$ or to a vertex of $G_{2}$. Now, for each such vertex $v$, we need to consider the specific graph $H \in \mathcal{H}$, such that the connected component of $G[X]$ containing $v$ is isomorphic to $H$. Moreover, we need to consider the position of $v$ in the copy of $H$, and a partition of the remaining vertices of $H$ between $G_{1}$ and $G_{2}$. However, since $\mathcal{H}$ is a fixed family of fixed graphs, the total number of recursive calls is still $2^{\tilde{O}\left(n^{2 / 3}\right)}$. We skip the proof, as it does not bring any new insight and is technically involved.

Theorem 8 MAXIMUM INDUCED $\mathcal{H}$-PACKING on string graphs with $n$ vertices can be solved in time $2^{\tilde{O}\left(n^{2 / 3}\right)}$.

Finally, note that if $\mathcal{H}$ contains graphs with different numbers of vertices, then instead of maximizing the number of vertices in $X$, we can alternatively maximize the number of connected components of $G[X]$.

\section{Lower Bounds}

Rather surprisingly, the win-win for 3-COLORING abruptly ceases to work for $k$ COLORING for every $k \geqslant 4$. First, let us consider the LIST 4-COLORING. Following Kratochvíl and Matoušek [32], by PURE 2- DIR we denote graphs admitting a 2- DIR representation in which parallel segments do not intersect. Observe that such a graph is bipartite.

Theorem 9 LIST 4-COLORING of a PURE 2- DIR graph cannot be solved in time $2^{o(n)}$, even if each list has size at most 3, unless the ETH fails.

Proof Let $\Phi$ be a 3-SAT formula with $n$ variables $v_{1}, v_{2}, \ldots, v_{n}$ and $m$ clauses $C_{1}, C_{2}, \ldots, C_{m}$. By repeating some literals in a clause, we may assume that each clause contains exactly three literals. For a clause $C_{i}$, let $v_{1}^{i}, v_{2}^{i}, v_{3}^{i}$ denote the variables of $C_{i}$.

We construct a 2- DIR graph $G$ with lists $L$ of colors from $\{1,2,3,4\}$, such that $\Phi$ is satisfiable if and only if $G$ is list-colorable with respect to the lists $L$.

For each variable $v_{i}$, we introduce a horizontal segment called $x_{i}$. For each clause $C_{i}$ we introduce three vertical segments $y_{1}^{i}, y_{2}^{i}, y_{3}^{i}$, corresponding to $v_{1}^{i}, v_{2}^{i}$, and $v_{3}^{i}$, respectively. We arrange them in a grid-like way (see Fig. 1). One may observe that the intersection graph induced by those segments is a biclique. We set the lists of each $x_{i}$ to $\{1,2\}$ and the lists of each $y_{1}^{i}, y_{2}^{i}, y_{3}^{i}$ to $\{3,4\}$. 
Fig. 1 The arrangement of variable- and occurrence-segments in $G$
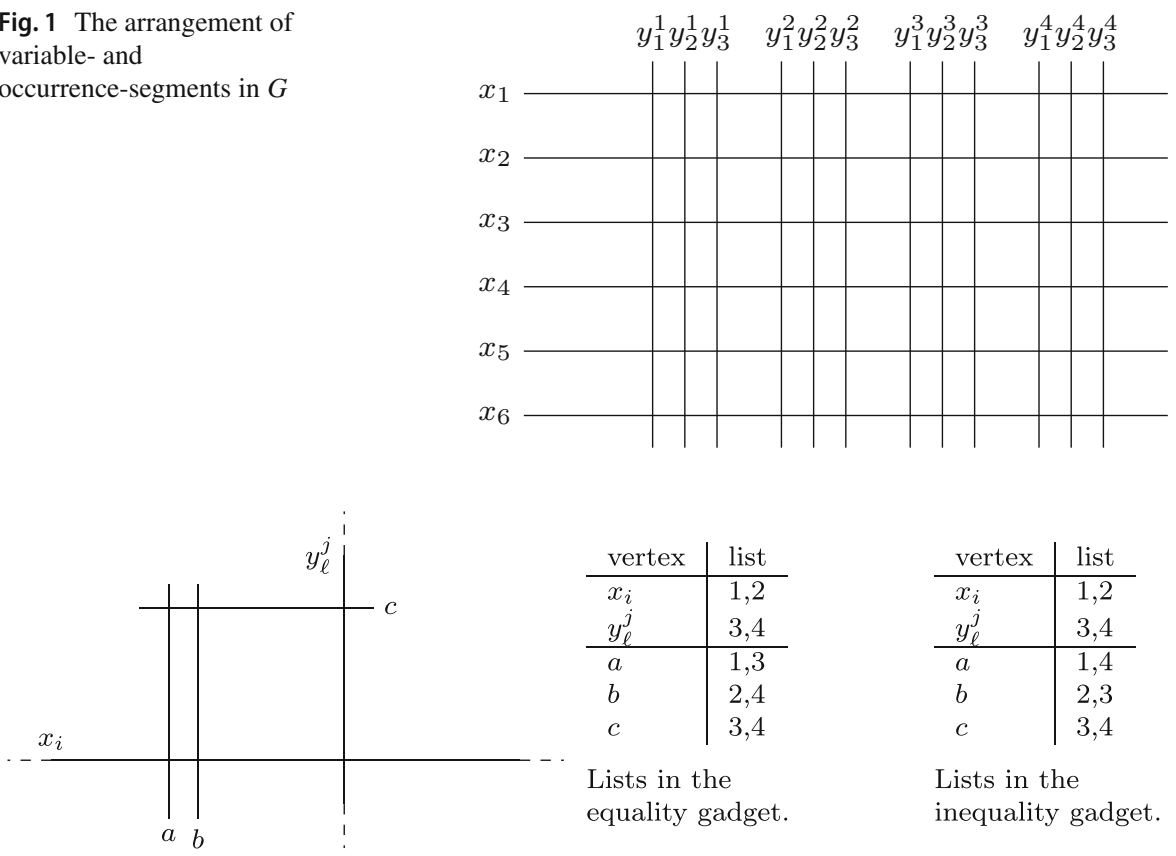

\begin{tabular}{l|l} 
vertex & list \\
\hline$x_{i}$ & 1,2 \\
$y_{\ell}^{j}$ & 3,4 \\
\hline$a$ & 1,3 \\
$b$ & 2,4 \\
$c$ & 3,4
\end{tabular}

Lists in the equality gadget.

\begin{tabular}{l|l} 
vertex & list \\
\hline$x_{i}$ & 1,2 \\
$y_{\ell}^{j}$ & 3,4 \\
\hline$a$ & 1,4 \\
$b$ & 2,3 \\
$c$ & 3,4
\end{tabular}

Lists in the inequality gadget.

Fig. 2 Equality and inequality gadgets. The arrangement of segments is the same in both gadgets, the only difference is the lists

The colors 1 and 2 used for $x_{i}$ will be interpreted, respectively, as true and false values given to $v_{i}$, while the colors 3 and 4 given to $y_{j}^{i}$ will be interpreted, respectively, as true and false values given to the literal corresponding to $v_{j}^{i}$.

To ensure this, we need to introduce equality gadgets and inequality gadgets. If the variable $v_{i}$ appears positively in the clause $C_{j}$ as its $\ell$-th literal, then at the crossing point of $x_{i}$ and $y_{\ell}^{j}$ we put the equality gadget ensuring that in any feasible coloring of $G$, the color of $x_{i}$ is 1 (2, respectively) if and only if the color of $y_{\ell}^{i}$ is 3 (4, respectively). On the other hand, if $v_{i}$ appears negatively in $C_{j}$ as its $\ell$-th literal, then at the crossing point of $x_{i}$ and $y_{\ell}^{j}$ we put the inequality gadget ensuring that in any feasible coloring of $G$, the color of $x_{i}$ is 1 (2, respectively) if and only if the color of $y_{\ell}^{j}$ is 4 (3, respectively).

The equality gadget consists of 3 segments, arranged as depicted on Fig. 2 and uses lists from the middle table on Fig. 2. Suppose $x_{i}$ gets the color 1. Then $a$ receives color 3 , and $c$ gets the color 4 . Thus the only choice for the color for $y_{\ell}^{j}$ is 3 . The coloring can be extended by coloring $b$ to 2 . The other cases are symmetric. The inequality gadget is analogous and uses the lists on the right side of Fig. 2.

The only thing left is to ensure that the coloring of $y_{1}^{j}, y_{2}^{j}, y_{3}^{j}$ exists if and only if $C_{j}$ is satisfied. This is ensured by the satisfiability gadget depicted in Fig. 3, attached to the top ends of $y_{j}^{1}, y_{2}^{j}$, and $y_{3}^{j}$. Note that the gadget can be colored if and only if at least one of $y_{1}^{j}, y_{2}^{j}, y_{3}^{j}$ gets color 3 , which is equivalent to one literal of $C_{j}$ being set to true. 


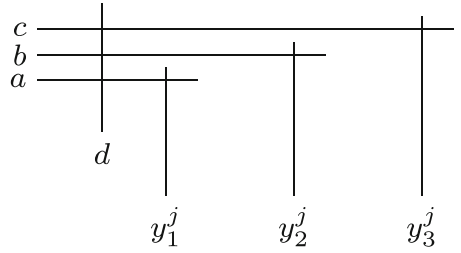

\begin{tabular}{l|l} 
vertex & list \\
\hline$y_{\ell}^{j}$ & 3,4 \\
\hline$a$ & 1,4 \\
$b$ & 2,4 \\
$c$ & 3,4 \\
$d$ & $1,2,3$
\end{tabular}

Fig. 3 Satisfiability gadget

The number of vertices of $G$ is $n^{\prime}=\underbrace{n}_{x_{i}}+\underbrace{3 m}_{y_{\ell}^{j}}+\underbrace{9 m}_{\text {(in)equality }}+\underbrace{4 m}_{\text {satisfiability }}=\Theta(n+$ $m)$. On the other hand, an algorithm solving list coloring of $G$ in time $2^{o\left(n^{\prime}\right)}$ can be used to decide the satisfiability of $\Phi$ in time $2^{o\left(n^{\prime}\right)}=2^{o(n+m)}$, which in turn contradicts the ETH.

The non-list version is obtained analogously to the hardness for 6-COLORING in [5]. We include the proof to make the paper self-contained.

Theorem 10 For every fixed $k \geqslant 4$, the $k$-COLORING problem of a 2-DIR graph cannot be solved in time $2^{o(n)}$, unless the ETH fails.

Proof We modify the construction from the proof of Theorem 9. We first introduce $k$ overlapping segments $R_{1}, R_{2}, \ldots, R_{k}$, whose coloring will serve as a reference coloring. Since these segments are pairwise intersecting, each of them receives a different color. We will denote by $i \in[k]$ the color assigned to $R_{i}$.

Now, for each segment $v$ of $G$, we want to simulate the list $L(v)$ from the instance of LIST 4-COLORING constructed in the proof of Theorem 9. For every color $i \notin L(v)$, we want to introduce a segment $s_{i}$ intersecting $v$, which will always receive color $i$.

To achieve this, we first need to transport the reference coloring to every gadget. We split it into two parts-we will separately transport colors 1 and 2, and colors greater than 2. The overall high-level idea is depicted in Fig. 4. Observe that this already simulates the lists for every $x_{i}$.

Such a construction relies on a constant-size gadget, which allows us to turn or split the reference coloring. The construction of this gadget is depicted in Fig. 5. Note that the number of segments in this gadget is constant if $k$ is constant. Moreover, turning or splitting the reference coloring of fewer than $k$ colors can be obtained by a simple adaptation of the turning gadget. Indeed, suppose we want to introduce a turning gadget for the set of colors $C \subseteq[k]$, with $|C|=k^{\prime}<k$ and we have $k^{\prime}$ overlapping segments carrying these colors. We introduce $k-k^{\prime}$ dummy segments, overlapping these segments. The dummy segments will clearly receive colors from $[k] \backslash C$, but we do not know which segment will get which color. Now we introduce a turning gadget for $k$ colors. We know which segments leaving the turning gadget get colors in $C$ (and we precisely know which segment gets which color). We do not need the remaining segments anymore, so we can finish them as soon as they leave the turning gadget.

Now, the only thing left is to connect every segment in every gadget to appropriate segments carrying the reference coloring (note that each $y_{\ell}^{j}$ belongs to some 


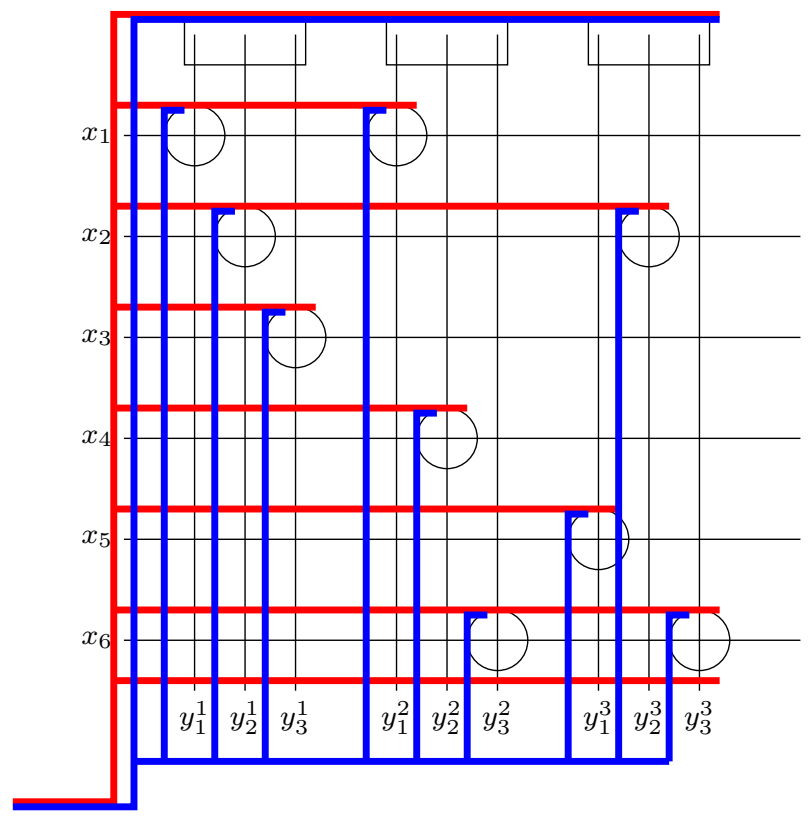

Fig. 4 Reference coloring is transported to every gadget. Circles denote the (in)equality gadgets, while rectangles denote the satisfiability gadgets. Red and blue lines denote, respectively, pairs of overlapping segments with colors 1,2 , and colors greater than 2 . Segments $R_{1}, R_{2}, \ldots, R_{k}$ are positioned in the lower left corner of the picture (Color figure online)

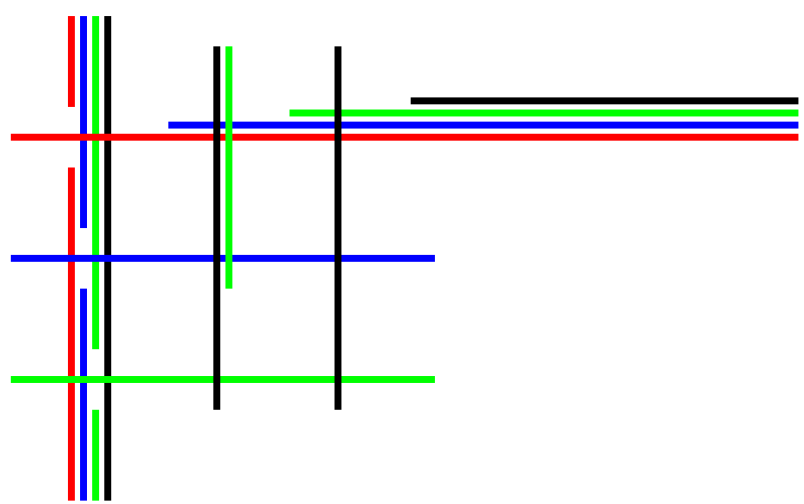

Fig. 5 Turning gadget for $k=4$ colors. The parallel segments depicted close to each other are overlapping. Observe that the depicted 4-coloring is the only possible (up to the permutation of colors). For $k>4$ the turning gadget is analogous (Color figure online)

(in)equality gadget). This can easily be done using a constant number of additional segments per gadget: we introduce a turning gadget for $k$ colors, and finish the segments that are not needed anymore before they intersect the segments in gadgets (see Fig. 6). 

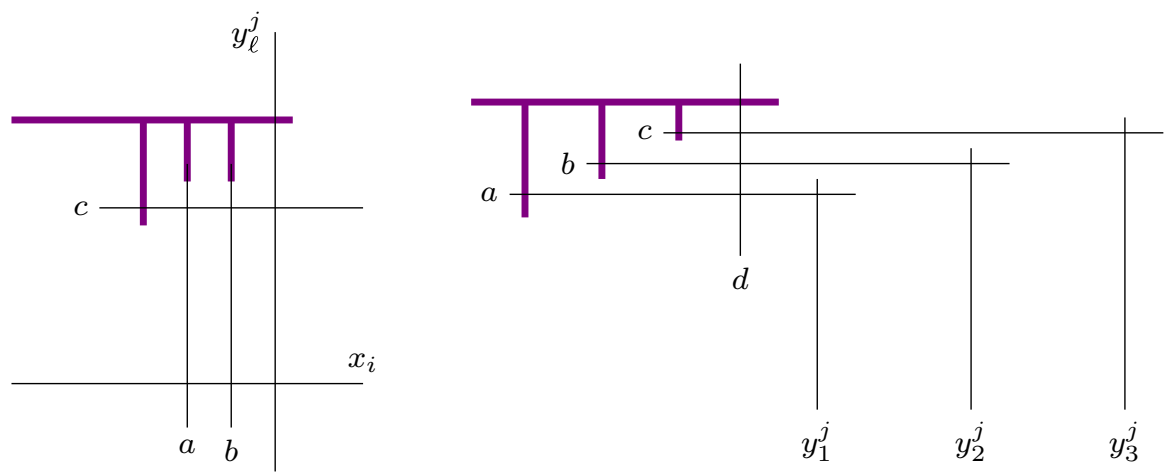

Fig. 6 Simulation of lists for vertices in (in)equality gadgets and satisfiability gadgets. Horizontal violet lines denote tuples of overlapping segments, carrying the reference coloring (all $k$ colors). In the vertical lines, segments carrying unnecessary colors are finished before they intersect the segments of the gadgets (Color figure online)

The total size of the construction increases by a constant factor, as we introduce $O(n)$ constant-size turning gadgets. Thus an algorithm for $k$-coloring the constructed 2- DIR graph in time $2^{o\left(n^{\prime}\right)}$ could be used to solve any 3- SAT instance in time $2^{o(n)}$, refuting the ETH.

Observe that the construction in the proof of Theorem 9 cannot be performed with segments of bounded lengths, since segments $x_{i}$ and $y_{k}^{j}$ need to have length $O(n)$ (while the segments inside the gadgets can have unit length). For unit segments, we show the following weaker lower bound.

Theorem 11 For every fixed $k \geqslant 4$, LIST $k$-COLORING of a unit 2- DIR graph or $k$ COLORING of a unit 3- DIR cannot be solved in time $2^{o\left(n^{2 / 3}\right)}$, unless the ETH fails.

Proof Consider a 3- SAT instance with variables $v_{1}, v_{2}, \ldots, v_{n}$ and clauses $C_{1}, C_{2}, \ldots$, $C_{m}$, where $m=\Theta(n)$. By duplicating some literals if necessary, we may assume that each clause contains exactly three literals.

Let us start with a reduction to LIST $k$-COLORING in unit 2- DIR. For each clause $C_{j}$ we introduce three $d$ isjoint vertical unit segments, each corresponding to one literal in $C_{j}$. These segments will be called literal segments related to $C_{j}$. We place them in such a way that the distance between the leftmost and the rightmost literal segment (of all literal segments) is slightly smaller than $1 / 2$. The ordering of the segments is the following: first, the literal segments related to the clause $C_{1}$, then literal segments related to the clause $C_{2}$ and so on. Moreover, they are slightly shifted vertically, so that the $y$-coordinates of their top endpoints form an increasing sequence. We set the list of possible colors for each literal segment to $\{3,4\}$ and for each three literal segments related to a single clause, we introduce a satisfiability gadget already shown in Fig. 3. We will interpret 3 as assigning the value true to the particular literal (and 4 will correspond to false). Figure 7 shows the placement of literal segments and satisfiability gadgets. Analogously, for each variable $v$, we introduce a vertical segment for each occurrence of $v$ (we call these segments occurrence segments) with list $\{3,4\}$. The segments are placed in such a way that the distance between the leftmost and the 


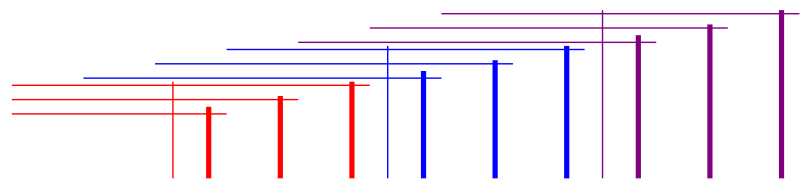

Fig. 7 Placement of satisfiability gadgets. Segments in one color correspond to one clause. Literal segments are depicted by thick lines. Note that all segments can be freely extended to the left and to the bottom, to make them unit (Color figure online)

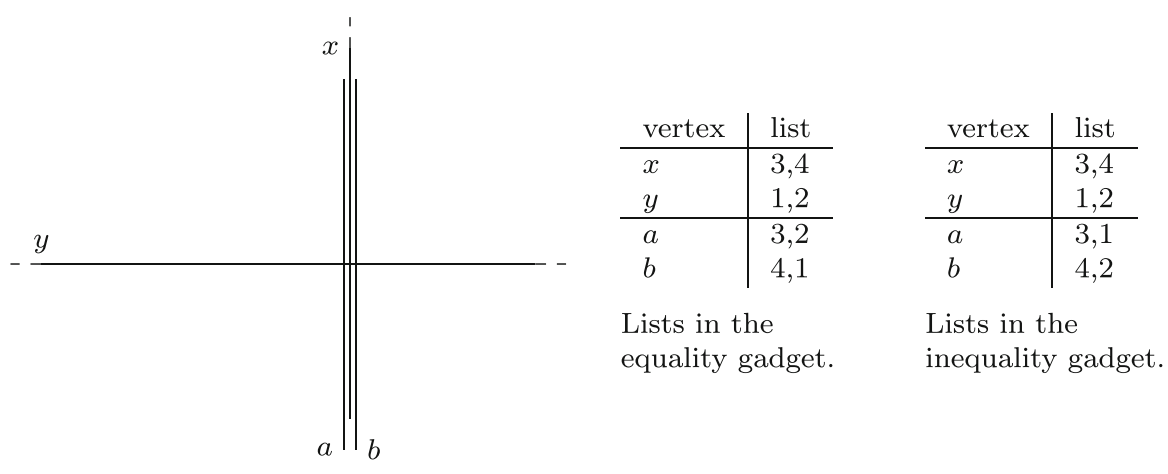

Fig. 8 Modified equality and inequality gadgets. The segment $x$ is an occurrence segment and the segment $y$ is a variable segment. Segments $a, b$, and $x$ overlap

rightmost occurrence segment is slightly smaller than $1 / 2$. Moreover, leftmost occurrence segments correspond to $v_{1}$, then we put the segments for $v_{2}$ etc. For a variable $v_{i}$ for $i \in[n]$, we introduce a horizontal segment, intersecting the occurrence segments of variables $v_{i}, v_{i+1}, \ldots, v_{n}$. These segments will be called variable segments. The variable segments are pairwise disjoint and each has list $\{1,2\}$. Again, we will interpret 1 as the value true given to a variable, and 2 will denote false. Now we need to ensure that the truth assignment defined by the coloring of occurrence segments is consistent. For this, we will use a slightly modified version of the (in)equality segment introduced in Fig. 2. The modified gadget is shown in Fig. 8. For a variable $v$ and its positive occurrence, we introduce an equality gadget joining the occurrence segment and the variable segment. Analogously, we introduce an inequality gadget joining the variable segment and the occurrence segment corresponding to a negative occurrence. The placement of all these segments is shown in Fig. 9.

Now we need to make sure that the truth assignment given by the coloring of literal segments is consistent with the truth assignment given by the coloring of occurrence segments. We will do it in a very similar way as we synchronized the colorings of occurrence segments, i.e., by using auxiliary horizontal segments and equality gadgets. Let $\ell_{1}, \ell_{2}, \ldots, \ell_{3 m}$ denote the literals ordered as their corresponding literal segments (from left to right). Let $o_{1}, o_{2}, \ldots, o_{3}$ be the ordering of occurrences, again from left to right. Let $\sigma$ be the permutation of $[3 m]$, such that the literal $\ell_{i}$ corresponds to the occurrence $o_{\sigma(i)}$. Now, for every $i \in[n]$, we want to introduce an equality gadget between the literal segment corresponding to $\ell_{i}$ and the occurrence segment corresponding to $o_{\sigma(i)}$. 


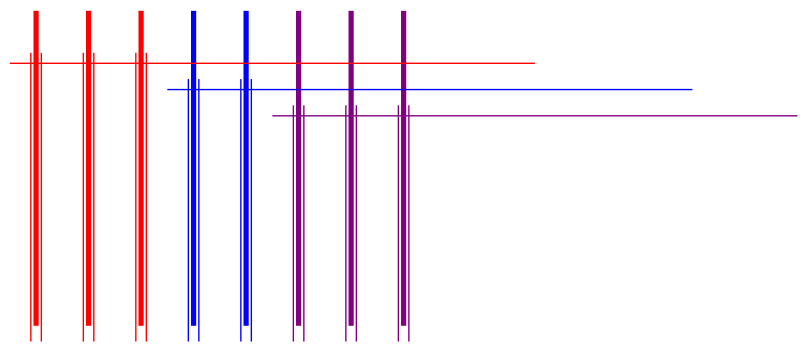

Fig. 9 Placement of variable and occurrence segments along with (in)equality gadgets. Segments in one color correspond to one variable. Literal segments are depicted by thick lines. Thin horizontal lines are variable segments and thin vertical lines are parts of (in)equality gadgets (Color figure online)

We observe that this is quite easy to do if $\sigma$ is either increasing or decreasing. It follows from basic properties of Young tableaux (see Knuth [29, Sec. 5.1.4]) that each permutation of [3m] can be partitioned into $z \leqslant\lceil\sqrt{6 m+1 / 4}-1 / 2\rceil=O(\sqrt{n})$ monotone sequences, and this partition can be found in polynomial time (see also Brandstädt and Kratsch [10]). Let $\sigma_{1}, \sigma_{2}, \ldots, \sigma_{z}$ be the partition of $\sigma$, where each $\sigma_{i}$ is monotone. We introduce $z$ layers, each corresponding to one $\sigma_{i}$. The $i$ th layer is responsible for synchronizing the colorings of literal segments and occurrence segments corresponding to elements of $\sigma_{i}$ (we call such segments important for layer $i$ ). Figure 10 shows a single layer, we set the distance between horizontal segments to be very small, compared to the length of each segment. Moreover, the horizontal segments cross the vertical literal and occurrence segments near their middle points. Note that each layer contains copies of all literal segments and occurrence segments. They appear in two groups-literal segments on the left and occurrence segments on the right. The distance between leftmost and rightmost segment in one group is slightly less than $1 / 2$, and the distance between the rightmost literal segment and the leftmost occurrence segment is slightly less than 2 . This allows us to fit two equality gadgets, whose horizontal segments are collinear but non-overlapping (see Fig. 10 and notice that we can adjust the distances within groups and between the groups, so that the distance between the horizontal segments is always positive and smaller than 1). For each such pair we introduce $k-1$ overlapping segments with lists $\{1,2, \ldots, k\}$, intersecting both of them and nothing else.

The last thing to do is to connect the literal segments (with (in)equality gadgets), layers, and occurrence segments with satisfiability gadgets. We place the occurrence segments at the bottom, and then we introduce layers in such a way that the corresponding vertical segments are collinear but non-intersecting. Finally, the literal segments with satisfiability gadgets are put on top (see Fig. 12 left).

Now consider two consecutive layers $i$ and $i+1$. Let $s_{1}$ and $s_{2}$ be collinear vertical segments and let $s_{1}$ be above $s_{2}$ (i.e., $s_{1}$ is in the layer $i+1$, and $s_{2}$ is in the layer $i$, see Fig. 11). Let us assume that $\sigma_{i}$ is increasing, the other case is symmetric. Observe that the top end of $s_{2}$ is not covered by (i.e., does not belong to) any other segment introduced so far. If $s_{1}$ is an important literal segment of the $i$ th layer, then there are two segments $a, b$ belonging to the appropriate equality gadget, which are covering the lower endpoint of $s_{1}$. We introduce $k-3$ overlapping vertical segments 


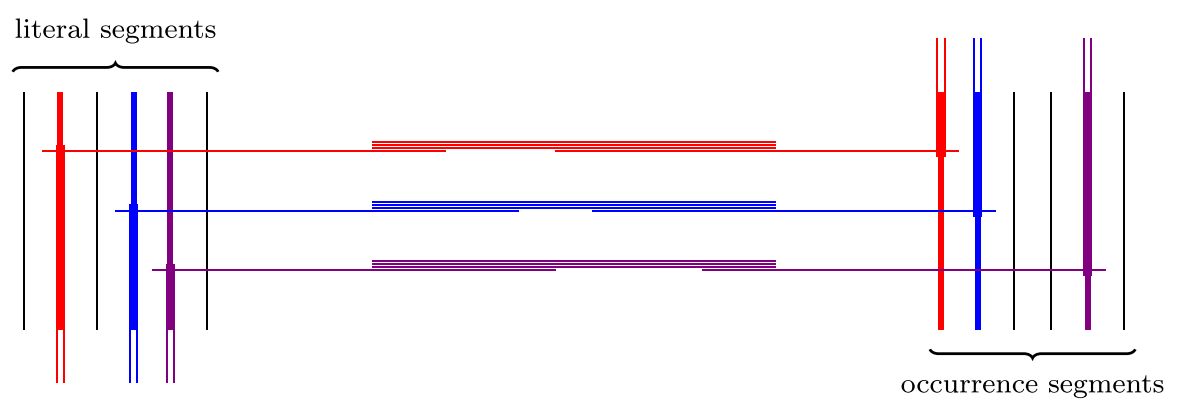

Fig. 10 A single layer $i$. Thick colored segments indicate corresponding literal and occurrence segments, which are important for layer $i$. Thin colored segments are parts of equality gadgets (Color figure online)

$q_{1}, q_{2}, \ldots, q_{k-3}$, intersecting only $s_{1}, a, b$ (so its upper endpoint is below the horizontal segment of the equality gadget, where $a$ and $b$ belong), and $s_{2}$, and set their lists to $\{1,2, \ldots, k\}$. The distance between the layers can be adjusted do that these newly introduced segments have no other intersections. Indeed, this is possible since the horizontal segments in each layer cross the vertical ones near their middle points.

If $s_{1}$ is not an important segment (and $s_{2}$ is important or not), we introduce $k-1$ overlapping segments $q_{1}, q_{2}, \ldots, q_{k-1}$ with lists $\{1,2, \ldots, k\}$, intersecting $s_{1}, s_{2}$, and no other previously constructed segment. This way $s_{1}$ and $s_{2}$ are non-adjacent, but they are both intersected by $k-1$ pairwise intersecting segments. This ensures that $s_{1}$ and $s_{2}$ will receive the same color.

In an analogous way we synchronize the colorings of occurrence segments, however this time segments from equality gadgets cover top ends of important occurrence segments.

The number of segments in each layer is $O(n)$ and the number of layers is $z=$ $O(\sqrt{n})$. Thus the total number of segments in our construction is $O\left(n^{3 / 2}\right)$. This implies that an algorithm solving LIST $k$-COLORING on unit 2-DIR graphs with $N$ vertices in time $2^{o\left(N^{2 / 3}\right)}$ could be used to solve 3 - SAT with $n$ variables in time $2^{o(n)}$, which contradicts the ETH.

If we want to obtain a reduction to the non-list $k$-COLORING for any $k \geqslant 4$, we need to transport the reference coloring to each gadget. However, this cannot be done for segments $a, b$ in our (in)equality gadgets (recall Theorem 10 and Fig. 8), which have non-trivial lists and are fully covered by other segments-note that if a segment in color 4 intersects $a$, it will also intersect $x$ (note that the segments with lists $\{1,2, \ldots, k\}$, even if they are fully covered, are not problematic since they do not need the reference coloring). But if we use a third direction, we can make $a, b$ intersect $x$ and $y$ (again, we use notation in Fig. 8) and no other vertex with non-trivial list in our grid-like structure-it is enough to choose their slope to be "almost vertical" (see Fig. 12, right). Now we can transport the reference coloring just as we did in the proof of Theorem 10, see Fig. 4. This construction adds a linear number of segments per layer, which is $O\left(n^{3 / 2}\right)$ segments in total.

This shows the claimed lower bound, for every fixed $k \geqslant 4$, for k-COLORING of unit 3- DIR graphs and completes the proof. 


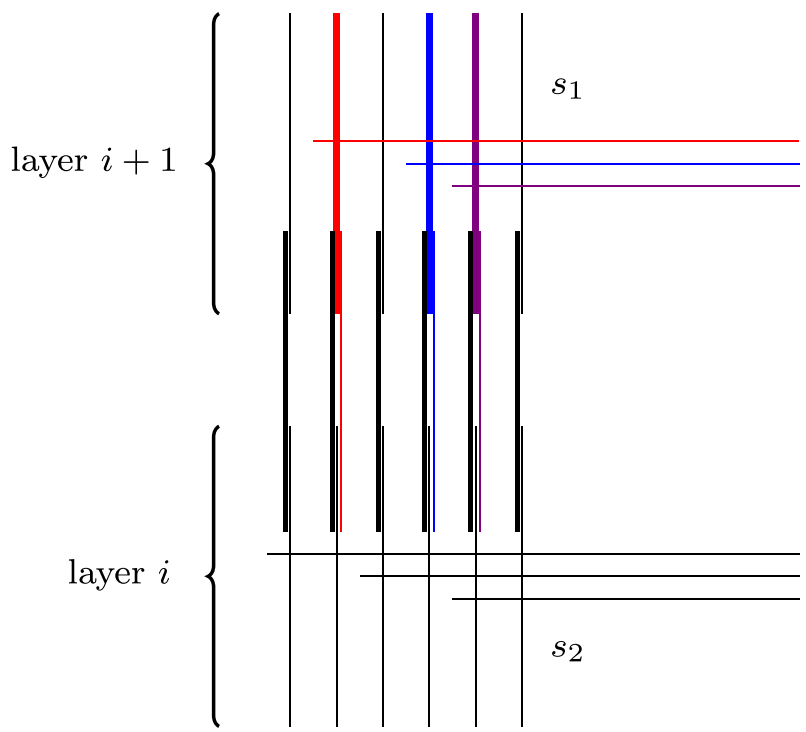

Fig. 11 Literal segments of two consecutive layers $i$ and $i+1$. Thick colored segments indicate segments that are important in the given layer. Thin colored segments are parts of equality gadgets (they denote overlapping segments $a$ and $b$. Thick black segments are bundles of segments $q_{i}$ (Color figure online)
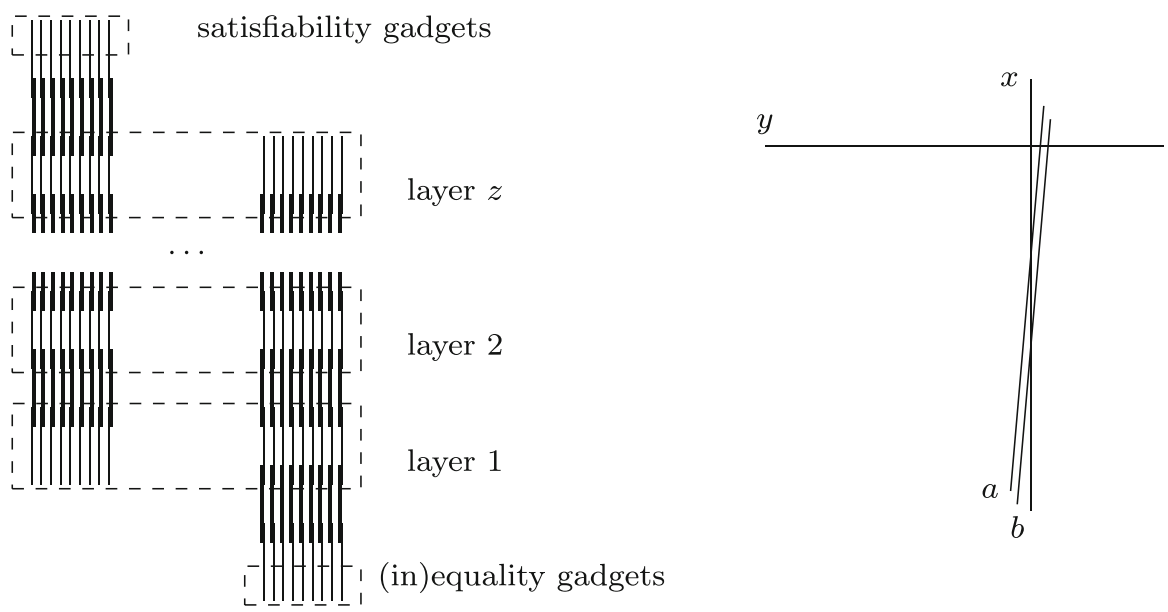

Fig. 12 Left: overall construction. Right: modified equality gadgets in a unit 3- DIR graph

It is perharps interesting to mention that for any choice three distinct directions, the class of intersection graphs of segments parallel to these three directions is the same [12]. However, this is no longer the case if we consider unit segments only. In particular, the choice of the three directions of segments in the construction from Theorem 11 depends on the initial 3- SAT instance. In other words, we cannot perform the construction with any three prescribed directions. 
Now we show that on segment graphs, Min Dominating Set, Min Connected Dominating Set, and Min IndEPENDENT DominAting SeT are unlikely to have a subexponential algorithm.

Theorem 12 MIN (CONNECTED) DOMINATING SET cannot be solved in time $2^{o(n)}$ on segment graphs with $n$ vertices, unless the ETH fails.

Proof By introducing new variables and clauses, we can transform an arbitrary 3- SAT formula $\psi$ with $N^{\prime}$ variables and $M^{\prime}=O\left(N^{\prime}\right)$ clauses into an equivalent CNF- SAT formula $\phi$ with $N=O\left(N^{\prime}+M^{\prime}\right)$ variables and $M=O\left(N^{\prime}+M^{\prime}\right)$ clauses, where each variable appears exactly twice positively and twice negatively. Indeed, say a variable $x_{i}$ appears $t$ times, one can create $t$ copies $x_{i}^{1}, x_{i}^{2}, \ldots, x_{i}^{t}$ and equate them by the chain of implications $\neg x_{i}^{1} \vee x_{i}^{2}, \neg x_{i}^{2} \vee x_{i}^{3}, \ldots \neg x_{i}^{t-1} \vee x_{i}^{t}, \neg x_{i}^{t} \vee x_{i}^{1}$. Now, each occurrence of $x_{i}$ is replaced by a different $x_{i}^{s}$. To use all the copies of the chain of implications exactly once more positively and negatively, one can add equivalent literals to a clause initially containing $x_{i}$ or $\neg x_{i}$.

Put $M$ pairwise-disjoint small segments on a circle as shown in Fig. 13; $M$ slightly perturbed points work as long as no two pairs define the same direction. Each small segment $s\left(C_{j}\right)$ represents a distinct clause $C_{j}$. For each literal $\sigma x_{i}$, where $x_{i}$ is one of the $N$ variables appearing in $\phi$ and $\sigma \in\{\emptyset, \neg\}$, we add a segment $s\left(\sigma x_{i}\right)$ crossing only the two small segments $s\left(C_{j}\right)$ and $s\left(C_{j^{\prime}}\right)$ corresponding to the clauses this literal satisfies. We extend all the segments corresponding to literals to make them pairwise intersect.

For each pair of literals $x_{i}, \neg x_{i}$, we add a small segment $s(i)$ near the intersection of $s\left(x_{i}\right)$ and $s\left(\neg x_{i}\right)$ which intersects only $s\left(x_{i}\right)$ and $s\left(\neg x_{i}\right)$. This finishes the construction (see Fig. 13). Note that the total number of segments is $n:=3 N+M=\Theta(N)$. We claim that there is a dominating set of size $N$ in the intersection graph if and only if $\phi$ is satisfiable.

Indeed, to dominate all the segments $s(i)$ for $i \in[N]$, one has to take at least one of $s\left(x_{i}\right)$ and $s\left(\neg x_{i}\right)$, hence exactly one of them since the sought after dominating set should have size $N$. The choice of the literals will only dominate all the segments $s\left(C_{j}\right)$ for $j \in[M]$ if the chosen dominating set corresponds to a satisfying assignment. The reverse direction is straightforward.

Moreover, as every pair of segments representing literals intersects, the dominating set encoding the satisfiable assignment is connected (it even induces a clique).

The hardness proof for Min InDEPEndEnt Dominating SET has to be quite different and is trickier.

Theorem 13 MIN INDEPENDENT DOMINATING SET cannot be solved in time $2^{o(n)}$ on segment graphs with $n$ vertices, unless the ETH fails.

Proof We reduce from 3-SAT on $N$ variables and $M$ clauses, where each variable appears at most four times, and each clause contains exactly three literals. This restriction was shown NP-complete by Tovey [46] via a linear reduction, so the ETH lower bound holds for this problem too. We can further assume that each literal appears at most three times. Indeed, if the same literal appears four times, then by definition its 
Fig. 13 An example with 9 clauses and 6 variables. The segments $s\left(x_{i}\right)$ and $s\left(\neg x_{i}\right)$ can be inferred from $s(i)$ or from the colors (although we only specified which is which for variable $\left.x_{1}\right)$. Observe that the light blue (i.e., connecting $s\left(C_{5}\right)$ to $s\left(C_{7}\right)$, and $s\left(C_{3}\right)$ to $\left.s\left(C_{9}\right)\right)$ and purple (connecting $s\left(C_{3}\right)$ to $s\left(C_{5}\right)$, and $s\left(C_{2}\right)$ to $\left.s\left(C_{7}\right)\right)$ pairs do not intersect inside the circle so those segments should be extended outside it until they meet (this part is not shown in the picture) (Color figure online)

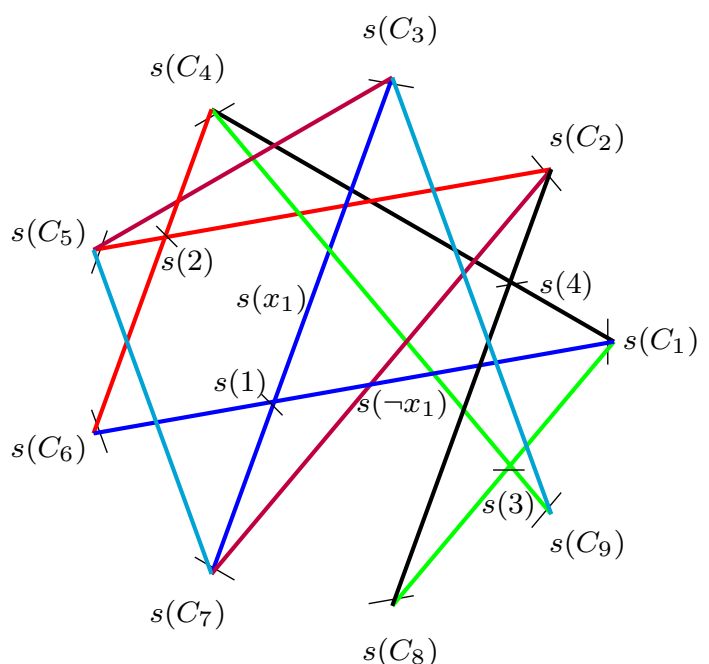

negation cannot appear in the whole instance. Hence, the variable can be set so as to satisfy the four corresponding clauses.

The variable gadget $G\left(x_{i}\right)$ for variable $x_{i}$ consists of three parallel segments $T_{i}$ representing positive occurrences crossing three parallel segments $F_{i}$ representing negative occurrences. Those two sets of segments intersect three dummy pairs of parallel segments as shown on Fig. 14. Note that even if a literal appears strictly fewer than three times, we keep exactly three parallel segments to encode it.

The clause gadget $G\left(C_{j}\right)$ for the clause $C_{j}$ consists of three pairwise crossing segments, drawn in blue in Fig. 14, each of which crosses one red segment. Each pair of blue and red segment corresponds to one of literals of $C_{j}$, these segments will be called literal segments. Additionally, all blue segments are intersected by four parallel dummy segments, and each pair of crossing blue and red segment has a private segment, crossing both of them.

Now, for every literal $x_{i}\left(\neg x_{i}\right)$ belonging to the clause $C_{j}$, we add a new segment denoted by $s\left(x_{i}, C_{j}\right)$ or $s\left(\neg x_{i}, C_{j}\right)$, respectively. We call these segments occurrence segments. This segment crosses one non-dummy segment in $G\left(x_{i}\right)$ : a segment of $T_{i}$ if the literal is positive, and a segment of $F_{i}$ otherwise. Moreover, it crosses exactly one red literal segment from $G\left(C_{j}\right)$, and no other segments in variable and clause gadgets, see Fig. 15. Each red literal segment is crossed by exactly one occurrence segment, and each segment of $\bigcup_{i \in[n]} T_{i} \cup F_{i}$ is crossed by at most one occurrence segment.

We claim that such a constructed graph has an independent dominating set of size at most $3 N+3 M$ if and only if the initial formula is satisfiable.

First, suppose that $A$ is a satisfying assignment. If $x_{i}$ is set to true by $A$, we select the three segments of $T_{i}$ in the solution, otherwise we select the three segments of $F_{i}$. Now consider a clause $C_{j}$. Since $A$ is satisfying, it contains at least one true literal. In $G\left(C_{j}\right)$ we select the blue segment corresponding to a true literal and the red segments corresponding to the other two literals. Note that this way we select $3 N+3 M$ segments and the selected set dominates all segments from all gadgets. Let us consider 
Fig. 14 The variable gadget $G\left(x_{i}\right)$ for the variable $x_{i}$ (left) and the clause gadget $G\left(C_{j}\right)$ for the clause $C_{j}$ (right)

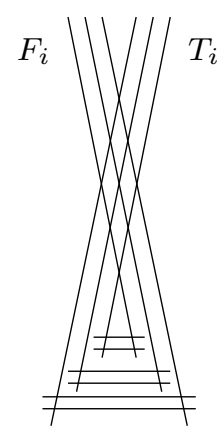

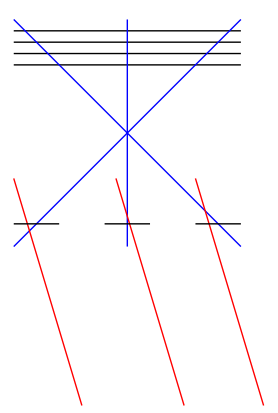

an occurrence segment $s\left((\neg) x_{i}, C_{j}\right)$. Either it is dominated by one of the selected red segments in $G\left(C_{j}\right)$, or it corresponds to a true literal of $C_{j}$, so it is dominated by a selected segment in $G\left(x_{i}\right)$.

On the other hand, assume that there is an independent dominating set $S$ of size at most $3 N+3 M$. Notice that in order to dominate all dummy segments in $G\left(x_{i}\right)$, we need to select at least three segments from $G\left(x_{i}\right)$, and if we want to select exactly three, we need to choose either all segments in $T_{i}$, or all segments in $F_{i}$. Analogously, we need to select at least three segments from each $G\left(C_{j}\right)$, and if we want to select exactly three, we need do choose exactly one of blue segments and thus we cannot choose its corresponding red segment. Note that since the total size of $S$ is $3 N+3 M$, we need to select exactly three segments in each gadget, and no occurrence segment is selected.

We define the assignment $A$ as follows: if segments $T_{i}$ are in $S$, then $x_{i}$ is set true, and otherwise $x_{i}$ is set false. Suppose that $C_{j}$ is not satisfied by $A$, which means that all its literals are false. This means that the three occurrence segments joining appropriate variable gadgets with $G\left(C_{j}\right)$ are not dominated by segments in variable gadgets, so $S$ must contain all three red segments from $G\left(C_{j}\right)$. However, this way the horizontal segments from $G\left(C_{j}\right)$ are not dominated, a contradiction.

The total number of segments is bounded by $12 N+13 M+3 M=O(N+M)$, so the claim holds.

Theorem 14 MAX CLIQUE cannot be solved in time $2^{o(n)}$ on string graphs with $n$ vertices, unless the ETH fails.

Proof We reduce from 3-SAT with a linear number of clauses, where every clause contains exactly three literals. Let $\phi$ be an instance with $N$ variables and $M=\Theta(N)$ clauses. For any positive integers $p$ and $s$, the co-cluster $\mathrm{CC}_{p, s}=K_{s, s, \ldots, s \text { (p times) }}$ can be represented as in Fig. 16.

We encode the $N$ variables by $2 N$ curves representing true and false for each variable by a co-cluster $\mathrm{CC}_{N, 2}$ and the $M=\Theta(N)$ clauses by $3 M$ curves each representing a distinct literal in a clause by a co-cluster $\mathrm{CC}_{M, 3}$. There are in total $n:=2 N+3 M=\Theta(N)$ curves. We make the $2 N$ variable curves intersect the $3 M$ literal curves in a grid-like way. They form an almost complete biclique $K_{2 N, 3 M}$ where $3 M$ edges are removed. 


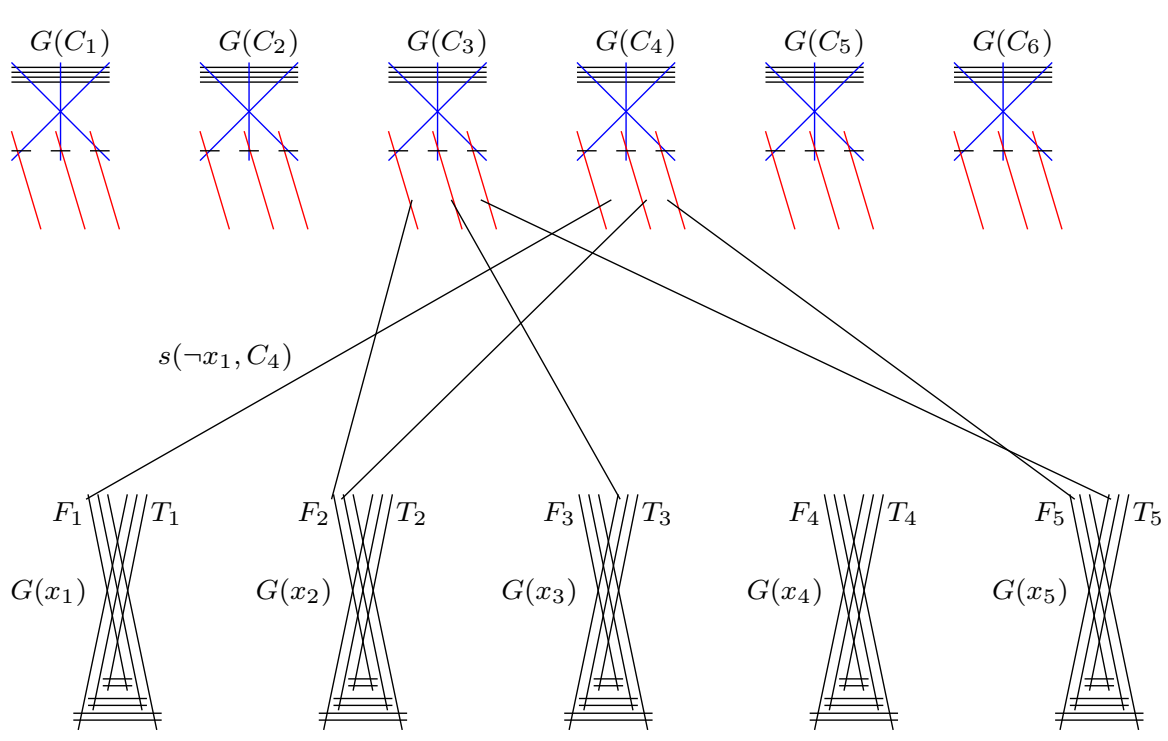

Fig. 15 The overall picture. We only represented two clauses: $C_{3}=\neg x_{2} \vee x_{3} \vee x_{5}$ and $C_{4}=\neg x_{1} \vee \neg x_{2} \vee$ $\neg x_{5}$

Fig. 16 Realization of a co-cluster $\mathrm{CC}_{p, s}=K_{s, s, \ldots, s}$ with $s=3$ and $p=7$

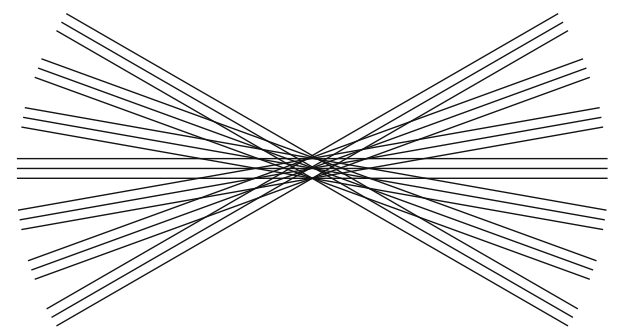

More precisely, the literal curve $c\left(l_{i}^{j}\right)(j \in[M], i \in[3])$ intersects every variable curve but $c\left(\sigma x_{k}\right)(k \in[N])$ encoding the $k$-th variable with sign $\sigma \in\{\emptyset, \neg\}$ for which $l_{i}^{j}$ and $\sigma x_{k}$ are opposite literals (see Fig. 17).

It is easy to observe that there is a clique of size $N+M$ if and only if $\phi$ has a satisfying assignment.

\section{Perspectives}

We have started a generalized optimality program on segment and string graphs for the most principal graph problems. On the algorithmic side, we extended a subexponential algorithm for MAX INDEPENDENT SET on string graphs [22] to some other problems, namely 3-Coloring, Min FEedBack VerTeX SET, and MAXIMUM INDUCED MATCHING. On the complexity side, we showed that no subexponential algorithm is likely for, among others, 4-Coloring, Min DominAting SET, and Min INDEPENDENT DOMINATING SET. It is quite easy to obtain such lower bounds for string graphs. 


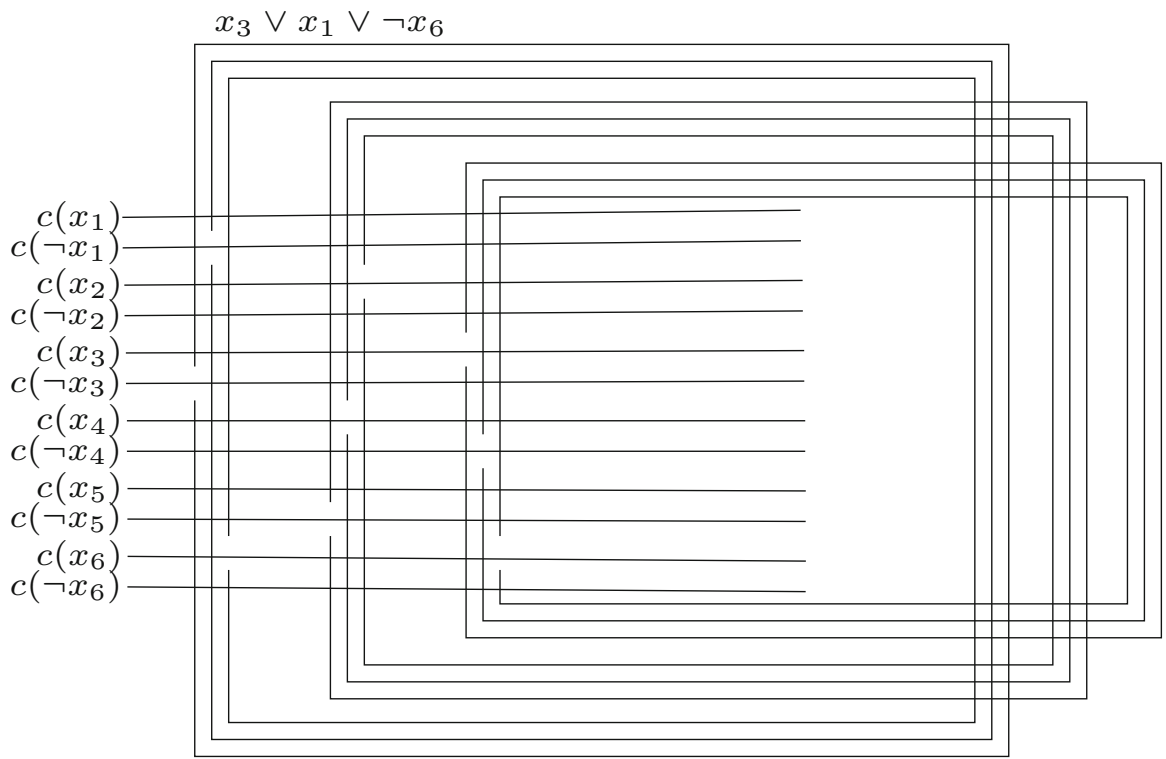

Fig. 17 The representation of 3 clauses: $x_{3} \vee x_{1} \vee \neg x_{6}, x_{5} \vee \neg x_{4} \vee \neg x_{2}$, and $\neg x_{3} \vee x_{4} \vee \neg x_{6}$

Extending those results to segments requires more ingenuity, and even more so when it comes to unit segments.

A handful of questions remains unsettled. Can we improve the algorithm or give tight ETH lower bounds for the following problems: MAX INDEPENDENT SET without geometric representation, 3-COLORING, and Min FEEDBACK VERTEX SET on segments/strings? Can we show for MAX CLIQUE the same lower bound for segment graphs as we have for string graphs. The mere NP-hardness of MAX CLIQUE on segments [11] answered a long-standing open question. Hence, it is likely that getting a tight ETH hardness will be difficult. We would also find it interesting to have, for a certain problem, an algorithm for segments (resp. unit segments) which beats the ETH lower bound on strings (resp. segments). So far, we only have candidate problems for such a "separation".

Finally, another natural continuation of this work is to determine which fixedparameter tractable problems can be solved in time $O^{*}\left(2^{\tilde{O}\left(k^{2 / 3}\right)}\right)$ or $O^{*}\left(2^{\tilde{O}(\sqrt{k})}\right)$, and which W[1]-hard problems can be solved in time $f(k) n^{O(\sqrt{k})}$ on segments and strings. For instance, MIN VERTEX COVER can be solved in time $O^{*}\left(2^{\tilde{O}\left(k^{2 / 3}\right)}\right)$ (even in time $O^{*}\left(2^{\tilde{O}(\sqrt{k})}\right)$ if a geometric representation is given with $O^{*}\left(2^{\tilde{O}(\sqrt{k})}\right)$ intersections) on string graphs due to the linear kernel yielding an equivalent instance on $2 k$ vertices and the algorithm for MAX INDEPENDENT SET. The latter problem can be solved in $n^{O(\sqrt{k})}$ in segments or more generally in polygons of polynomial complexity [36], while Min DOMinating SET on string graphs cannot be solved in time $f(k) n^{o(k)}$, for any computable function $f$, unless the ETH fails (since this lower bound holds for split graphs). 
Open Access This article is distributed under the terms of the Creative Commons Attribution 4.0 International License (http://creativecommons.org/licenses/by/4.0/), which permits unrestricted use, distribution, and reproduction in any medium, provided you give appropriate credit to the original author(s) and the source, provide a link to the Creative Commons license, and indicate if changes were made.

\section{References}

1. Adamaszek, A., Har-Peled, S., Wiese, A.: Approximation schemes for independent set and sparse subsets of polygons. CoRR, arXiv:1703.04758 (2017)

2. Adamaszek, A., Wiese, A.: A QPTAS for maximum weight independent set of polygons with polylogarithmically many vertices. In: Proceedings of the Twenty-Fifth Annual ACM-SIAM Symposium on Discrete Algorithms, SODA 2014, Portland, Oregon, USA, January 5-7, 2014, pp. 645-656 (2014)

3. Alber, J., Fiala, J.: Geometric separation and exact solutions for the parameterized independent set problem on disk graphs. J. Algorithms 52(2), 134-151 (2004)

4. Baker, B.S.: Approximation algorithms for NP-complete problems on planar graphs. J. ACM 41(1), 153-180 (1994)

5. Biró, C., Bonnet, É., Marx, D., Miltzow, T., Rzążewski, P.: Fine-grained complexity of coloring unit disks and balls. In: 33rd International Symposium on Computational Geometry, SoCG 2017, July 4-7, 2017, Brisbane, Australia, pp. 18:1-18:16 (2017)

6. Bonnet, É., Brettell, N., Kwon, O., Marx, D.: Generalized feedback vertex set problems on boundedtreewidth graphs: chordality is the key to single-exponential parameterized algorithms. In: Lokshtanov, D., Nishimura, N. (eds.) 12th International Symposium on Parameterized and Exact Computation, IPEC 2017, September 6-8, 2017, Vienna, Austria, volume 89 of LIPIcs, pp. 7:1-7:13. Schloss DagstuhlLeibniz-Zentrum fuer Informatik (2017)

7. Bonnet, É., Brettell, N., Kwon, O., Marx, D.: Generalized feedback vertex set problems on boundedtreewidth graphs: chordality is the key to single-exponential parameterized algorithms. CoRR, arXiv:1704.06757 (2017)

8. Bonnet, É., Rzążewski, P.: Optimality program in segment and string graphs. In: Brandstädt, A., Köhler, E., Meer, K. (eds.) Graph-Theoretic Concepts in Computer Science-44th International Workshop, WG 2018, Cottbus, Germany, June 27-29, 2018, Proceedings, volume 11159 of Lecture Notes in Computer Science, pp. 79-90. Springer (2018)

9. Boyer, J.M., Myrvold, W.J.: On the cutting edge: simplified $O(n)$ planarity by edge addition. J. Graph Algorithms Appl. 8(2), 241-273 (2004)

10. Brandstädt, A., Kratsch, D.: On partitions of permutations into increasing and decreasing subsequences. Elektronische Informationsverarbeitung und Kybernetik 22(5/6), 263-273 (1986)

11. Cabello, S., Cardinal, J., Langerman, S.: The clique problem in ray intersection graphs. Discrete Comput. Geom. 50(3), 771-783 (2013)

12. Cerný, J., Král, D., Nyklová, H., Pangrác, O.: On intersection graphs of segments with prescribed slopes. In: Mutzel, P., Jünger, M., Leipert, S. (eds.) Graph Drawing, 9th International Symposium, GD 2001 Vienna, Austria, September 23-26, 2001, Revised Papers, volume 2265 of Lecture Notes in Computer Science, pp. 261-271. Springer (2001)

13. Chalopin, J., Gonçalves, D.: Every planar graph is the intersection graph of segments in the plane: extended abstract. In: Proceedings of the 41st Annual ACM Symposium on Theory of Computing, STOC 2009, Bethesda, MD, USA, May 31-June 2, 2009, pp. 631-638 (2009)

14. Demaine, E.D., Fomin, F.V., Hajiaghayi, M.T., Thilikos, D.M.: Bidimensional parameters and local treewidth. SIAM J. Discrete Math. 18(3), 501-511 (2004)

15. Demaine, E.D., Fomin, F.V., Hajiaghayi, M.T., Thilikos, D.M.: Subexponential parameterized algorithms on bounded-genus graphs and $H$-minor-free graphs. J. ACM 52(6), 866-893 (2005)

16. Demaine, E.D., Hajiaghayi, M.: The bidimensionality theory and its algorithmic applications. Comput. J. 51(3), 292-302 (2008)

17. Demaine, E.D., Hajiaghayi, M.: Linearity of grid minors in treewidth with applications through bidimensionality. Combinatorica 28(1), 19-36 (2008) 
18. Demaine, E.D., Hajiaghayi, M.T.: Fast algorithms for hard graph problems: bidimensionality, minors, and local treewidth. In: GD 2014 Proceedings, pp. 517-533 (2004)

19. Erdős, P., Szekeres, G.: A Combinatorial Problem in Geometry, pp. 49-56. Birkhäuser, Boston (1987)

20. Fomin, F.V., Lokshtanov, D., Panolan, F., Saurabh, S., Zehavi, M.: Finding, hitting and packing cycles in subexponential time on unit disk graphs. CoRR, arXiv:1704.07279 (2017)

21. Fomin, F.V., Lokshtanov, D., Saurabh, S.: Bidimensionality and geometric graphs. In: Rabani, Y., (ed.) Proceedings of the twenty-third annual ACM-SIAM symposium on discrete algorithms, SODA 2012, Kyoto, Japan, January 17-19, 2012, pp. 1563-1575. SIAM (2012)

22. Fox, J., Pach, J.: Computing the independence number of intersection graphs. In: Proceedings of the Twenty-Second Annual ACM-SIAM Symposium on Discrete Algorithms, SODA 2011, San Francisco, California, USA, January 23-25, 2011, pp. 1161-1165 (2011)

23. Fox, J., Pach, J.: Applications of a new separator theorem for string graphs. CoRR, arXiv:1302.7228 (2013)

24. Fox, J., Pach, J., Tóth, C.D.: A bipartite strengthening of the crossing lemma. J. Comb. Theory Ser. B 100(1), 23-35 (2010)

25. Gonçalves, D., Isenmann, L., Pennarun, C.: Planar graphs as L-intersection or L-contact graphs. In: Czumaj, A., (ed) Proceedings of the Twenty-Ninth Annual ACM-SIAM Symposium on Discrete Algorithms, SODA 2018, New Orleans, LA, USA, January 7-10, 2018, pp. 172-184. SIAM (2018)

26. Har-Peled, S.: Quasi-polynomial time approximation scheme for sparse subsets of polygons. In: 30th Annual Symposium on Computational Geometry, SOCG'14, Kyoto, Japan, June 08-11, 2014, pp. 120 (2014)

27. Impagliazzo, R., Paturi, R.: On the complexity of $k$-SAT. J. Comput. Syst. Sci. 62(2), 367-375 (2001)

28. Impagliazzo, R., Paturi, R., Zane, F.: Which problems have strongly exponential complexity? J. Comput. Syst. Sci. 63(4), 512-530 (2001)

29. Knuth, D.E.: The Art of Computer Programming, Sorting and Searching, vol. III. Addison-Wesley, Boston (1973)

30. Kratochvíl, J.: String graphs. II. Recognizing string graphs is NP-hard. J. Comb. Theory Ser. B 52(1), 67-78 (1991)

31. Kratochvíl, J., Matoušek, J.: String graphs requiring exponential representations. J. Comb. Theory Ser. B 53(1), 1-4 (1991)

32. Kratochvíl, J., Matoušek, J.: Intersection graphs of segments. J. Comb. Theory Ser. B 62(2), 289-315 (1994)

33. Lee, J.R.: Separators in region intersection graphs. CoRR, arXiv:1608.01612 (2016)

34. Lipton, R.J., Tarjan, R.E.: Applications of a planar separator theorem. SIAM J. Comput. 9(3), 615-627 (1980)

35. Marx, D.: Parameterized complexity of independence and domination on geometric graphs. In: Parameterized and Exact Computation, Second International Workshop, IWPEC 2006, Zürich, Switzerland, September 13-15, 2006, Proceedings, pp. 154-165 (2006)

36. Marx, D., Pilipczuk, M.: Optimal parameterized algorithms for planar facility location problems using Voronoi diagrams. In: Bansal, N., Finocchi, I. (eds) ESA 2015 Proceedings, volume 9294 of LNCS, pp. 865-877. Springer (2015)

37. Marx, D., Pilipczuk, M.: Optimal parameterized algorithms for planar facility location problems using Voronoi diagrams. CoRR, arXiv:1504.05476 (2015)

38. Matoušek, J.: Intersection graphs of segments and $\exists \mathbb{R}$. CoRR, arXiv:1406.2636 (2014)

39. Matoušek, J.: Near-optimal separators in string graphs. Comb. Probab. Comput. 23(1), 135-139 (2014)

40. McDiarmid, C., Müller, T.: Integer realizations of disk and segment graphs. J. Comb. Theory Ser. B 103(1), 114-143 (2013)

41. Robertson, N., Seymour, P.D., Thomas, R.: Quickly excluding a planar graph. J. Comb. Theory Ser. B 62(2), 323-348 (1994)

42. Schaefer, M., Sedgwick, E., Štefankovič, D.: Recognizing string graphs in NP. J. Comput. Syst. Sci. 67(2), 365-380 (2003)

43. Schaefer, M., Štefankovič, D.: Fixed Points, Nash Equilibria, and the existential theory of the reals. Theory Comput. Syst. 60(2), 172-193 (2017)

44. Scheinerman, E.: Intersection classes and multiple intersection parameters of graphs. PhD thesis, Princeton University (1984)

45. Smith, W.D., Wormald, N.C.: Geometric separator theorems and applications. In: FOCS 1998 Proceedings, pp. 232-243, Washington, DC, USA, 1998. IEEE Computer Society 
46. Tovey, C.A.: A simplified NP-complete satisfiability problem. Discrete Appl. Math. 8(1), 85-89 (1984)

47. Zverovich, I.E., Zverovich, V.E.: An induced subgraph characterization of domination perfect graphs. J. Graph Theory 20(3), 375-395 (1995)

Publisher's Note Springer Nature remains neutral with regard to jurisdictional claims in published maps and institutional affiliations. 